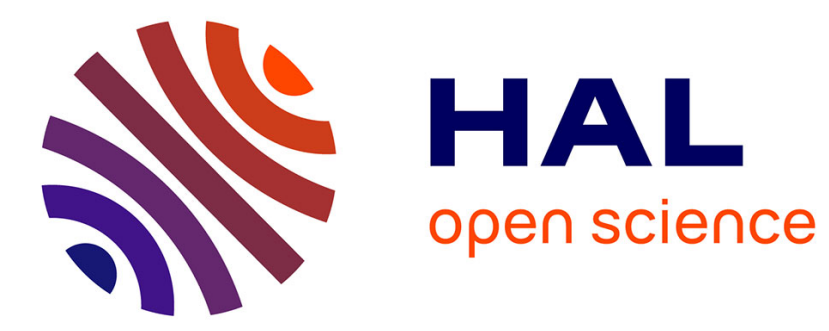

\title{
Neural anatomy of echinoid early juveniles and comparison of nervous system organization in echinoderms
}

Laurent Formery, François Orange, Antoine Formery, Shunsuke Yaguchi, Christopher Lowe, Michael Schubert, Jenifer C. Croce

\section{To cite this version:}

Laurent Formery, François Orange, Antoine Formery, Shunsuke Yaguchi, Christopher Lowe, et al.. Neural anatomy of echinoid early juveniles and comparison of nervous system organization in echinoderms. Journal of Comparative Neurology, 2021, 529, pp.1135-1156. 10.1002/cne.25012 . hal02929239

\section{HAL Id: hal-02929239 \\ https://hal.science/hal-02929239}

Submitted on 19 Nov 2020

HAL is a multi-disciplinary open access archive for the deposit and dissemination of scientific research documents, whether they are published or not. The documents may come from teaching and research institutions in France or abroad, or from public or private research centers.
L'archive ouverte pluridisciplinaire HAL, est destinée au dépôt et à la diffusion de documents scientifiques de niveau recherche, publiés ou non, émanant des établissements d'enseignement et de recherche français ou étrangers, des laboratoires publics ou privés. 


\section{Neural anatomy of echinoid early juveniles and comparison of nervous system organization in echinoderms}

Short running title: Echinoderm neural anatomy

Laurent Formery ${ }^{1}$, François Orange ${ }^{2}$, Antoine Formery ${ }^{3}$, Shunsuke Yaguchi ${ }^{4}$, Christopher J. Lowe ${ }^{5}$, Michael Schubert ${ }^{1}$ Jenifer C. Croce ${ }^{1^{*}}$

1. Laboratoire de Biologie du Développement de Villefranche-sur-Mer (LBDV), Evolution of Intracellular Signaling in Development (EvoInSiDe), Sorbonne Université, CNRS, Villefranche-sur-Mer, France.

2. Centre Commun de Microscopie Appliquée (CCMA), Université Côte d'Azur, Nice, France.

3. Freelance 3D Modelization, Paris, France.

4. Shimoda Marine Research Station, University of Tsukuba, Shizuoka, Japan.

5. Department of Biology, Hopkins Marine Station, Stanford University, Pacific Grove, California, USA.

*Corresponding author: Jenifer C. Croce, Laboratoire de Biologie du Développement de Villefranche-sur-Mer (LBDV), UMR7009, Institut de la Mer de Villefranche (IMEV), 181 Chemin du Lazaret, 06230 Villefranche-sur-Mer, France.

Email: jenifer.croce@obs-vlfr.fr, ORCID ID: https://orcid.org/0000-0001-7092-0257

Data Availability Statement: The authors confirm that the data supporting the findings of this study are available within the article and its supplementary material.

Conflict of Interest: The authors declare no conflict of interest.

Author's contribution: Laurent Formery, Michael Schubert, and Jenifer C. Croce designed the study and wrote the manuscript with input from all authors. Laurent Formery performed experiments, acquired confocal images, performed data analyses, and generated all figures. François Orange conducted the scanning electron microscopy experiments. Antoine Formery 
created the 3D images. Shunsuke Yaguchi supervised part of the work on echinoids. Christopher J. Lowe oversaw the work on holothuroids and asteroids. Jenifer C. Croce supervised most of the work on echinoids, contributed to data analyses, and submitted the manuscript. All authors approved the final version of the manuscript.

Funding: French Ministry of Research and Technology, Japan Society for the Promotion of Science, André Picard Network, National Aeronautics and Space Administration. 


\section{Abstract}

The echinoderms are a phylum of marine deuterostomes characterized by the pentaradial (five fold) symmetry of their adult bodies. Due to this unusual body plan, adult echinoderms have long been excluded from comparative analyses aimed at understanding the origin and evolution of deuterostome nervous systems. Here, we investigated the neural anatomy of early juveniles of representatives of three of the five echinoderm classes: the echinoid Paracentrotus lividus, the asteroid Patiria miniata, and the holothuroid Parastichopus parvimensis. Using whole mount immunohistochemistry and confocal microscopy, we found that the nervous system of echinoid early juveniles is composed of three main structures: a basiepidermal nerve plexus, five radial nerve cords connected by a circumoral nerve ring, and peripheral nerves innervating the appendages. Our whole mount preparations further allowed us to obtain thorough descriptions of these structures and of several innervation patterns, in particular at the level of the appendages. Detailed comparisons of the echinoid juvenile nervous system with those of asteroid and holothuroid juveniles moreover supported a general conservation of the main neural structures in all three species, including at the level of the appendages. Our results support the previously proposed hypotheses for the existence of two neural units in echinoderms: one consisting of the basiepidermal nerve plexus to process sensory stimuli locally and one composed of the radial nerve cords and the peripheral nerves constituting a centralized control system. This study provides the basis for more in-depth comparisons of the echinoderm adult nervous system with those of other animals, in particular hemichordates and chordates, to address the long-standing controversies about deuterostome nervous system evolution.

\section{Keywords}

Comparative anatomy, evolution, juvenile, sea urchin, sea cucumber, sea star. 


\section{Introduction}

The echinoderms are a large phylum of marine invertebrates. They comprise about 7,300 extant species (Appeltans et al., 2012) grouped into five extant classes (Telford et al., 2014): the echinoids (sea urchins), the holothuroids (sea cucumbers), the asteroids (sea stars), the ophiuroids (brittle stars), and the crinoids (feather stars and sea lilies). Anatomically, adult echinoderms are characterized by four shared characters (or synapomorphies): a radial adult body plan that is usually pentaradial (five fold) (Smith, 2008), a hydraulic water vascular system used mainly for locomotion (Nichols, 1972), an endoskeleton made of calcium carbonate ossicles (or plates) (Hyman, 1955), and a unique type of connective tissue composed of mutable collagen (Wilkie, 1984, 2002).

Most echinoderms are defined, during their lifespan, by an indirect mode of development (Arnone, Byrne, \& Martinez, 2015; Hyman, 1955), even though direct modes of development have independently evolved many times within the phylum (Wray \& Raff, 1991). During indirect development, the zygote first gives rise to a planktonic larva with bilateral symmetry. Midway through larval development, the pentaradial adult body plan then begins to develop on the left side of the larva as an imaginal rudiment. Once the rudiment is well developed, the larva undergoes metamorphosis, leading to the emergence of a benthic juvenile. Early juveniles resemble miniature versions of the adults, already possessing all adult echinoderm synapomorphies. However, immediately following metamorphosis, they sometimes lack a functional digestive tract, such as in echinoids and asteroids, in which the adult mouth and anus perforate only at later stages (Gosselin \& Jangoux, 1998; Haesaerts, Jangoux, \& Flammang, 2003). The endoskeleton of early juveniles is moreover rather transparent and their anatomy is relatively simple, since they have not yet initiated the characteristic metameric growth of echinoderms (Mooi, David, \& Wray, 2005). In addition, early juveniles are amenable to experimental investigations (Burke et al., 2006; Gao et al., 2015). Early juveniles have thus proven to be very useful experimental stand-ins for adult echinoderms.

Nervous systems represent critical components of animal body plans: they collect, process, and integrate sensory inputs from the body and the environment and coordinate appropriate responses. In echinoderms with indirect development, two distinct nervous systems develop during their lifecycle: one in the larva and one in the adult (Hinman \& Burke, 2018). The larval nervous system 
emerges during embryogenesis and is completely lost during metamorphosis (Bisgrove \& Raff, 1989; Chia \& Burke, 1978; Heinzeller \& Welsch, 2001). By contrast, the adult nervous system forms de novo in the rudiment, that is, independent of the larval one, and is maintained after metamorphosis (Dupont, Thorndyke, Thorndyke, \& Burke, 2009; Hirokawa, Komatsu, \& Nakajima, 2008; Katow, Elia, \& Byrne, 2009; Nakano, Murabe, Amemiya, \& Nakajima, 2006). The structure and development of echinoderm larval nervous systems have already been the subject of multiple anatomical and molecular investigations, in particular in echinoids (Angerer, Yaguchi, Angerer, \& Burke, 2011; Hinman \& Burke, 2018). In comparison, the adult nervous system of echinoderms has received much less attention. Most studies on echinoderm adult nervous systems have so far been focused on the histological characterization of their anatomy and ultrastructure (Hyman, 1955; Mashanov, Zueva, Rubilar, Epherra, \& Garcia-Arraras, 2016; Smith, 1965), with little characterization of the molecular details of their development or anatomy, compared to larvae (e.g., Adachi et al., 2018; Mashanov, Zueva, \& Garcia-Arraras, 2015; Mercurio, Gattoni, Messinetti, Sugni, \& Pennati, 2019; Sumner-Rooney, Rahman, Sigwart, \& Ullrich-Lüter, 2018; Tinoco et al., 2018). Furthermore, an integrated and comprehensive description of the neural organization of echinoderm adult nervous systems is still lacking, and it is still unclear to what extent this detailed organization is conserved across the different echinoderm classes.

Previous work has already reported that the adult nervous system of members of all five extant echinoderm classes is composed primarily of five radial nerve cords, joined together, at their proximal ends (i.e., in the vicinity of the mouth), by a circumoral nerve ring (Burke et al., 2006; Hyman, 1955; Smith, 1965). In addition, several other neural structures have been described in different echinoderm classes, including peripheral nerves emanating from the radial cords and innervating body musculature and appendages, peripheral ganglia located at the level of the appendages, and nerve plexuses innervating the epithelial lining of the viscera, the water vascular system and the body wall (Mashanov et al., 2016; Smith, 1965). Given these observations, some authors have hypothesized that the organization of the adult nervous systems of echinoderms is similar to that of chordates, which feature a central and a peripheral nervous system (Arshavskii, Kashin, Litvinova, Orlovskii, \& Fel'dman, 1976; Mashanov et al., 2016). In this scheme, the five radial 
nerve cords would act as a centralized integrative center capable of receiving peripheral sensory information and controlling behavioral outputs. However, other surveys have alternatively established that some peripheral structures of the echinoderm adult nervous system are capable of autonomous, local processing of sensory information (Bullock, 1965; Campbell, 1973; Kinosita, 1941). Consequently, other authors have advanced that adult echinoderms lack a bona fide integrative center and rely instead only on local information processing and output control (Cobb, 1987; Garm, 2017; Matsuzaka, Sato, Kano, Aonuma, \& Ishiguro, 2017).

Echinoderms, together with hemichordates, form the ambulacrarians, which along with the chordates constitute the deuterostomes (Cannon et al., 2014; Satoh, Rokhsar, \& Nishikawa, 2014). Although there is little doubt that the last common ancestor of chordates featured a centralized nervous system (Holland, 2015a), the reconstruction of the ancestral deuterostome nervous system is much less straightforward (Holland, 2015b; Lowe, Clarke, Medeiros, Rokhsar, \& Gerhart, 2015). In particular, interpretations of hemichordate data have proven to be polarized (Kaul-Strehlow, Urata, Minokawa, Stach, \& Wanninger, 2015; Lowe et al., 2003; Nomaksteinsky et al., 2009), and the current lack of information on the evolutionary diversification of echinoderm nervous systems further complicates this issue (Formery, Schubert, \& Croce, 2019; Holland, 2015b; Nakano et al., 2006). Additional information on the basic architecture of the adult nervous system of key representatives of different echinoderm classes, involving contemporary imaging approaches and a broad selection of markers, is thus needed to establish a comparative framework and enable novel evolutionary inferences.

Here, we carried out an in-depth survey of the neural anatomy of early juveniles of the echinoid species Paracentrotus lividus. We performed confocal microscopy in combination with whole mount immunohistochemistry to define the architecture of their adult-like nervous system in relation to their endoskeleton and musculature. In addition, to allow comparisons between different echinoderm classes, we carried out similar analyses in postmetamorphic specimens of the asteroid species Patiria miniata and the holothuroid species Parastichopus parvimensis. In summary, we found that the nervous system of an early juvenile of $P$. lividus is composed, like its adult counterpart, of three main structural components: (a) a basiepidermal nerve plexus spanning the entire body wall, (b) five radial nerve cords joined together by a circumoral nerve ring, and (c) several peripheral nerves. 
Based on our 3D whole mount preparations, we further described the organization of these components, both at the level of the test and the appendages, and were able to identify the innervation patterns of most organs, including the masticatory apparatus, the podia and the spines. We propose that in echinoids, the basiepidermal nerve plexus establishes a first neural unit responsible for the integration of local signals, while the radial nerve cords and peripheral nerves form a second neural unit involved in centralized processing of neural information. Comparisons of the echinoid nervous system with those of asteroids and holothuroids further confirmed the conservation of a similar general neuroanatomical organization in these three echinoderm classes and revealed striking similarities in the innervation patterns of the podia and spines. We thus argue that, although echinoderms have long been ignored in comparative, evolutionary studies, the comparison of the conserved components of their nervous systems with those of other animals hold enormous potential for addressing long-standing questions, notably about the origin and diversification of deuterostome nervous systems.

\section{Materials and Methods}

\section{Animals}

Gravid P. lividus adults were collected in the bay of Villefranche-sur-Mer (France). Gravid $P$. miniata and P. parvimensis adults were obtained from Monterey Bay (California, USA). For P. lividus, gamete collection and fertilization were performed using 1 male and at least 3 different females, following standard procedures (Lepage \& Gache, 1989). Embryos and larvae were cultured at 18$20^{\circ} \mathrm{C}$ in natural, filtered seawater, collected at "Point B" in the bay of Villefranche-sur-Mer $\left(43^{\circ} 41 \mathrm{~N}\right.$ $\left.-07^{\circ} 19 \mathrm{E}\right)$, at a depth of $20 \mathrm{~m}$, and filtered using a $0.2 \mu \mathrm{m}$ mesh. Embryos and larvae were raised in $250 \mathrm{~mL}$ and $5 \mathrm{~L}$ beakers, respectively, with constant stirring until metamorphosis. Following fertilization, embryos were cultured at a density of 100 individuals/mL. After $48 \mathrm{hr}$ of development, the culture concentration was adjusted to 1 individual $/ \mathrm{mL}$. Starting at $48 \mathrm{hr}$ of development, water exchange and feeding were performed every Monday, Wednesday, and Friday. Two-thirds of the seawater was changed and the larvae were fed using a 1:1 mix of freshly grown cultures of the microalgae Dunaliela salina (strain MCCV020) and Chaetoceros gracilis (strain UTEX2658). From 
day 2 to day 10, the larvae were fed with a final concentration of 8.000 cells $/ \mathrm{mL}$ and from day 11 to metamorphosis with a final concentration of 16.000 cells $/ \mathrm{mL}$. The larvae started undergoing spontaneous metamorphosis in the beakers after roughly 25 days of development. At day 4 postmetamorphosis, the early juveniles (or 4-day-old early juveniles) were anesthetized and deciliated by a quick transfer into $2 x$ concentrated artificial seawater $(1.032 \mathrm{mM} \mathrm{NaCl}, 20 \mathrm{mM} \mathrm{KCl}, 22 \mathrm{mM}$ $\mathrm{CaCl}_{2} \cdot 2 \mathrm{H}_{2} \mathrm{O}, 68 \mathrm{mM} \mathrm{MgCl} \cdot 6 \mathrm{H}_{2} \mathrm{O}, 44 \mathrm{mM} \mathrm{MgSO} \cdot \cdot 7 \mathrm{H}_{2} \mathrm{O}, 20 \mathrm{mM}$ EPPS, adjusted to $\left.\mathrm{pH} 8.2\right)$. After 3 to 5 seconds, the early juveniles were removed from the solution and either transferred to natural, filtered seawater for further culturing, mounted in filtered seawater for imaging with a Zeiss Axio Imager 2 microscope, or fixed for scanning electron microscopy (SEM), immunohistochemistry or histology. For $P$. miniata and $P$. parvimensis, larval rearing was performed as described in lowe and Wray (2000). At the desired postmetamorphic developmental stages, specimens were either mounted in filtered seawater for imaging with a Zeiss Axio lmager 2 microscope or fixed for immunohistochemistry.

\section{Scanning electron microscopy}

For scanning electron microscopy (SEM), P. lividus specimens were fixed in a $2.5 \%$ glutaraldehyde solution in $0.1 \mathrm{M}$ sodium cacodylate buffer $(\mathrm{pH} 7.4)$ prepared in filtered, artificial seawater. They were then stored overnight at $4^{\circ} \mathrm{C}$. Specimens were subsequently rinsed three times in distilled water for 15 minutes at room temperature (RT). The samples were then dehydrated in a series of ethanol washes, 10 minutes each at RT, with increasing alcohol concentrations diluted in distilled water: $25 \%, 50 \%, 75 \%, 96 \%$, and finally three times at $100 \%$. After a final wash in hexamethyldisilazane (HMDS) (Carl Roth) for $5 \mathrm{~min}$ at RT, the samples were left overnight at RT to dry. Specimens were coated with platinum $(3 \mathrm{~nm})$ prior to observations. SEM analyses were performed with a Tescan Vega-3-XMU scanning electron microscope at an accelerating voltage of 5 kV.

\section{Histology}

For histology, deciliated 4-day-old early juveniles of $P$. lividus were fixed overnight at $4{ }^{\circ} \mathrm{C}$ in $4 \%$ paraformaldehyde prepared in natural, filtered seawater containing $10 \mathrm{mM}$ EPPS. Specimens were dehydrated in a series of ethanol washes prepared in $1 x$ phosphate-buffered saline (PBS) with 
increasing alcohol concentrations: $25 \%, 50 \%, 75 \%$, and $100 \%$. Each wash was performed for $1 \mathrm{hr}$ at RT. Dehydrated specimens were washed in butanol overnight at $4{ }^{\circ} \mathrm{C}$ and then embedded in paraffin wax. Transversal semi-thin sections $(5 \mu \mathrm{m})$ were generated using a hand-operated microtome. The sections were subsequently stained with hematoxylin and eosin. For this, two washes were carried out in $100 \%$ xylene (Sigma-Aldrich), for $10 \mathrm{~min}$ each at RT, to remove the wax. Then, the sections were rehydrated by passing them through a graded series of ethanol solutions prepared in distilled water $(100 \%, 90 \%, 70 \%, 50 \%$, and $0 \%)$. Each rehydration step was performed twice for $15 \mathrm{~min}$ at RT. The sections were subsequently stained in Mayer's hematoxylin (Sigma-Aldrich) for $3 \mathrm{~min}$ at RT and then washed for $10 \mathrm{~min}$ under running tap water. Thereafter, the sections were quickly rinsed with distilled water before being soaked in a $95 \%$ ethanol solution for $30 \mathrm{~min}$ at RT. Staining in $1 \%$ Eosin Y solution (Sigma-Aldrich) was performed for 15 seconds at RT. Staining was followed by two 5 min washes at RT in $95 \%$ ethanol and two 5 min washes at RT in $100 \%$ ethanol. The sections were then incubated in a solution of $100 \%$ xylene until mounting. Prior to mounting, the stained sections were placed under a fume hood to enable xylene evaporation. Upon xylene evaporation, the stained sections were mounted in Permount Mounting Medium (Fisher Scientific) and imaged using a Zeiss Axio Imager 2 microscope with differential interference contrast (DIC) optics.

\section{Immunohistochemistry}

For immunohistochemistry, $P$. lividus, $P$. miniata, and $P$. parvimensis early juveniles were fixed for $10 \mathrm{~min}$ at RT in $3.7 \%$ formaldehyde prepared in natural, filtered seawater. Specimens were washed four times in PBS containing 0.05\% Tween-20 (PBST). Pre-blocking was carried out by incubating the specimens for $1 \mathrm{hr}$ at RT in SuperBlock Blocking Buffer (Thermo Fisher Scientific) for P. lividus or in Bovine Serum Albumin (BSA) (Sigma Aldrich), at a final concentration of $10 \mathrm{mg} / \mathrm{mL}$, for $P$. miniata and $P$. parvimensis. The samples were subsequently incubated, overnight at $4^{\circ} \mathrm{C}$, with primary antibodies diluted in PBST plus $0.005 \mathrm{U} / \mu \mathrm{l}$ rhodamine phalloidin (Thermo Fisher Scientific) to co-stain F-actin-rich structures, such as muscles. The primary antibodies were: mouse anti-MSP130 (mesenchyme-specific cell surface glycoprotein-130, also called Id5) (McClay, Cannon, Wessel, Fink, \& Marchase, 1983) used at 1:2, mouse anti-synaptotagmin-B (Nakajima, Kaneko, Murray, \& Burke, 2004) used at 1:2, mouse anti-acetylated $\alpha$-tubulin (Sigma-Aldrich, Cat\# T6793, 
RRID:AB_477585) used at $1: 200$, and rabbit anti-serotonin (ImmunoStar, Cat\# 20080, RRID: AB_572263) used at 1:200. The primary antibodies were removed by three washes in PBST for 10 min each at RT. Specimens were then incubated for $1 \mathrm{hr}$ at RT with one of the two following secondary antibodies diluted 1:200 in PBST: Alexa Fluor 647 goat anti-mouse lgG H\&L (Abcam, Cat\# abi50115, RRID:AB_2687948) and Cy5 goat anti-rabbit lgG H\&L (Bethyl Cat\# A120-201C5, RRID:AB_10631421). Nuclear staining was performed for $10 \mathrm{~min}$ at RT using TO-PROI-lodide (Thermo Fisher Scientific) diluted 1:500 in PBST. Decalcification of the endoskeleton was carried out by five washes in $50 \mathrm{mM}$ EDTA diluted in PBST for $10 \mathrm{~min}$ each at RT (Peters, Price, \& Horowitz, 2005). Subsequently, the juveniles were rinsed three times in PBST for 5 min each at RT and incubated in a series of $20 \%, 40 \%, 60 \%$, and $80 \%$ fructose diluted in PBST to clarify the tissue (procedure adapted from Ke, Fujimoto, \& Imai, 2013). Each fructose wash was carried out for at least $\mathrm{l} \mathrm{hr}$ at RT. Clarified juveniles were mounted in $80 \%$ fructose diluted in PBST for observation and imaging, which were performed using a Leica SP8 confocal microscope for P. lividus and a Zeiss Zl confocal microscope for $P$. miniata and P. parvimensis. For each sample, series of optical sections were taken at a $\mathrm{z}$-step interval of $1-2 \mu \mathrm{m}$ and multichannel acquisitions were obtained by sequential imaging. Confocal optical sections were compiled into maximum intensity z-projections using lmage] version 1.440 (RRID: SCR_003070) (Schneider, Rasband, \& Eliceiri, 2012). For P. lividus, computed transversal views of definitive and juvenile spines were obtained through $3 \mathrm{D}$ rendering and surface segmentation using Imaris version 7.7.2 (Oxford Instruments, RRID:SCR_007370). Depth-encoded images were further generated using the Image] plugin "Temporal-Color Code", developed by Kota Miura and Johannes Schindelin. All obtained images were processed using Adobe Photoshop CS5 (Adobe Photoshop, RRID:SCR_014199). For 3D reconstructions, surface rendering was performed on the synaptotagmin-B and phalloidin maximum intensity z-projections using lmaris version 7.5.0 (Oxford Instruments, RRID:SCR_007370). The background was removed using a particle size threshold, and the obtained images were processed using Blender version 2.79 (Blender Foundation, RRID:SCR_008606).

\section{Results}




\section{External anatomy of Paracentrotus lividus early juveniles}

Four days after metamorphosis, early juveniles (or 4-day-old early juveniles) of $P$. lividus had, like their adult counterparts, a spherical body, composed of a central skeletal shell (the test) harboring several appendages in the form of spines, podia, and pedicellariae (Figure 1, Supporting Information Figure Sia). Along the oral-aboral axis, the body of an early juvenile can be subdivided into three distinct regions (Gosselin \& Jangoux, 1998): (i) the supracoronal region, which constitutes the aboral surface (directed away from the substrate), (ii) the coronal region, located at the midline, and (iii) the infracoronal region, which corresponds to the oral surface (Figure la). On the supracoronal region, two types of appendages were commonly observed: the juvenile spines and the pedicellariae (Figure la,b). The juvenile spines are short and immotile spines that are lost as development proceeds (Emlet, 2010). They displayed either a triradiate or a tetraradiate tip (Figure la,b, Supporting Information Figure Sib). The pedicellariae are appendages devoted to the protection of the animal against parasites and predators (Ghyoot, De Ridder, \& Jangoux, 1987). These appendages were composed of three movable jaws allowing the pedicellariae to be either closed or open (Figure la,b, Supporting Information Figure Sic,d).

The coronal region also featured juvenile spines, along with five groups of four definitive spines that alternated with five groups of three podia (Figure la-c). In contrast to juvenile spines, definitive spines are long, motile, and single-pointed spines that are maintained in the adult (Emlet, 2010). Within a given quartet, definitive spines were morphologically indistinguishable from each other. They were always arranged sequentially, in a similar pattern, along the oral-aboral axis of the animal, with one spine facing the oral side, two spines being positioned medially, and one spine facing the aboral side (Figure Ic, Supporting Information Figure Sie). In 4-day-old early juveniles, each group of podia was further organized into a single central primary podium and two lateral secondary podia located underneath the central primary podium (Figure ic, Supporting Information Figure Sif). While primary podia are transient organs that develop in the rudiment and are lost as development proceeds, secondary podia are permanent podia that emerge upon metamorphosis and are subsequently maintained in the adult. By definition, all podia developing after metamorphosis are thus secondary podia (David et al., 1995; Gosselin \& Jangoux, 1998; Morris, 2009). In 4-day-old early 
juveniles, primary and secondary podia were anatomically similar, with primary podia being slightly larger, since they developed earlier (Supporting Information Figure Sif). Both podia types were composed of a flexible stem, capable of extending and retracting, that was anchored on one side within the animal body and that terminated on the other side in a discoid, flattened structure (Supporting Information Figure Sla,f). This distal flattened structure has previously been called the disk or the sucker (Agca, Elhajj, Klein, \& Venuti, 2011; Burke, 1980; Santos, Gorb, Jamar, \& Flammang, 2005; Smith, 1937). We, however, chose here to call it the papilla (i.e., referring to a small protuberance of tissue) to avoid confusion with other anatomical structures or misleading (Hennebert, Santos, \& Flammang, 2012).

On the oral side, the infracoronal region was covered by a smooth epithelium, called the peristome (Figure Ic) (Hyman, 1955), which harbored a set of additional growing appendages: the buccal podia (Figure lc). The selective name of these secondary podia reflects their specific distribution around the edge of the mouth, which has yet to form in 4-day-old early juveniles (Burke, 1989; Gosselin \& Jangoux, 1998). Consistent with previous reports, we here confirmed the absence, at the developmental stage investigated, of a perforated mouth at the center of the peristome (Figure Ic) and of a perforated anus at the center of the supracoronal region (Figure lb). In echinoids, the pentaradial symmetry of the animal is further marked by the presence of five rays (or radii), each composed of two morphologically distinct areas: the ambulacrum, which is aligned with the primary podia, and the interambulacrum, which is located between two ambulacra (Mooi \& David, 2008). The distribution of the appendages in the coronal region reflected the pentaradial organization of the juvenile body (Figure Ic).

\section{Skeletal and muscular anatomy of Paracentrotus lividus early juveniles}

To obtain additional anatomical landmarks for subsequent analyses, we next investigated the skeletal and muscular anatomy of 4-day-old early juveniles of P. lividus (Figure 2, Supporting Information Figures S2-S4). The structure of the endoskeleton was studied using an antibody recognizing the mesenchyme-specific cell surface glycoprotein MSP-130, commonly used in echinoid larvae to label skeletogenic mesenchyme cells (McClay et al., 1983). In early juveniles, this antibody revealed both the outer and inner endoskeleton (Figure 2b,c, Supporting Information Figure S2). For 
instance, at the level of the test, the MSP-130 staining highlighted the ossicles (or plates) constituting the endoskeleton, including the 10 buccal plates distinguishable individually on the oral side (Figure $2 \mathrm{~b}, \mathrm{c})$. For the appendages, the staining further labeled the endoskeleton of both the juvenile and the definitive spines, of the pedicellariae, and of the podia (Figure $2 b, c)$. In the spines, the MSP-130 antibodies marked the endoskeleton throughout the shaft (Supporting Information Figure S2a-c). In the pedicellariae and podia, by contrast, the MSP-130 staining was limited, in the former, to a small region at the level of the stem (Supporting Information Figure S2d,e) and, in the latter, to a disk located within the papilla (Supporting Information Figure S2f-h). Lastly, within the juvenile, the MSP-130 staining also revealed several inner endoskeletal structures that were all associated chiefly with the masticatory apparatus of the animal (known as Aristotle's lantern), including the jaws and the teeth (Supporting Information Figure S2i-l).

The muscular anatomy of early juveniles of P. lividus was subsequently surveyed using a rhodamine-conjugated phalloidin probe that labels F-actin filaments, which are particularly enriched in muscle fibers. This staining highlighted several muscles distributed throughout the body (Figure 2d,e, Supporting Information Figures S3, S4). For instance, at the level of the test, the phalloidin staining revealed the presence of a network of thin muscular fibers, just underneath the shell, that were organized as mesenteries (Figure 2d). Similar to other animals, these mesenteries maintain the internal organization of the perivisceral cavity (Jensen, 1985; Ziegler et al., 2009) that, in early juveniles of $P$. lividus, was occupied chiefly by the digestive tract and the water vascular system (Supporting Information Figure S3a-d). In echinoids, the water vascular system is composed of several different structures, including the axial complex, the ring canal, the radial canals, the ampullae, and the podia (Ezhova et al., 2018; Ziegler et al., 2009). The phalloidin staining labeled the muscle fibers associated with all of these structures (Figure 2d,e, Supporting Information Figure S3cg). For instance, in the supracoronal region, it revealed the muscles associated with the most aboral portion of the axial complex (Figure 2d). In the coronal region, it further highlighted dense longitudinal muscles located within both the primary and the secondary podia (Figure 2e). Of note, these muscles in the podia were found running all along the stem, reaching the tip of the papilla, and extending through the central pore of the skeletal disk located within the papilla (Supporting Information Figure S3f,g). 
In the supracoronal region, two specific muscles were further found, which were associated with the pedicellariae (Figure 2d). These two muscles, called the adductor and abductor muscles (Peters \& Campbell, 1987), displayed a very characteristic, triangular shape (Supporting Information Figure S3h,i). In the coronal region, several muscle strands were also detected at the level of the definitive spines. These muscle strands were specifically located around the tubercle of the spines (Figure $2 \mathrm{~d}, \mathrm{e}$, Supporting Information Figure 3j,k). They constitute what we refer to as the ring muscle. By contrast, for the juvenile spines, we did not observe any associated muscular structure neither in the supracoronal nor in the coronal region (Figure 2d), which is consistent with their lack of motility.

Within the early juveniles of $P$. lividus, we also identified, in addition to the muscles of the water vascular system, several muscles associated with Aristotle's lantern (Figure 2e, Supporting Information Figure S4a-j). Previous studies, carried out on adults, have reported ten different muscles connected to the masticatory and respiratory functions of Aristotle's lantern (Stauber, 1993; Ziegler et al., 2012). Here, in 4-day-old early juveniles of P. lividus, we identified nine (Supporting Information Figure S4a-j). Lastly, a detailed examination of the infracoronal region highlighted several supplementary muscles embedded within the peristome and linked to the rim, lips, or jaws of the developing mouth (Supporting Information Figure S4k-m).

\section{General overview of the neuroanatomy of Paracentrotus lividus early juveniles}

To characterize the nervous system of 4-day-old early juveniles of P. lividus, we targeted three antigens by immunohistochemistry that are regularly used to study metazoan nervous systems: the neurotransmitter serotonin, the neuron-specific membrane protein synaptotagmin-B, and acetylated $\alpha$-tubulin, a specific posttranslationally modified form of tubulin present in axons and dendrites (Supporting Information Figure S5, Figure 3) (Black \& Keyser, 1987; Ferreira \& Cáceres, 1989; Gavilán, Perea-Atienza, \& Martínez, 2016; Nakajima et al., 2004; Richter et al., 2010; Zieger, Candiani, Garbarino, Croce, \& Schubert, 2018). Interestingly, for serotonin, we did not observe any specific signal in 4-day-old early juveniles (Supporting Information Figure S5a-b). Consistently, the adult rudiment of competent larvae of P. lividus was also devoid of serotonin staining, while the nervous system of the competent larvae was marked with a very conspicuous neural signal (Supporting Information Figure S5c). These observations indicate that the nervous system of young P. lividus 
adults lacks serotonergic neurons, a result that is consistent with previous reports from other echinoderms, such as the echinoid Hemicentrotus pulcherrimus, the ophiuroid Amphipholis kochii, and the holothuroid Stichopus japonicus (Hirokawa et al., 2008; Katow et al., 2009; Nakano et al., 2006).

Next, we used the synaptotagmin-B and acetylated $\alpha$-tubulin antibodies (Figure 3). The synaptotagmin-B antibody is known to label cellular processes and synaptic structures of most neurons in echinoderms (Nakajima et al., 2004). The acetylated $\alpha$-tubulin antibody is widely used in metazoans as a pan-neuronal marker labeling neurites (Richter et al., 2010; Zieger et al., 2018), although it also labels cilia (Arikawa \& Williams, 1993). The combination of the two antibodies provided a comprehensive view of the juvenile nervous system of $P$. lividus (Figure 3), with the caveat that neuronal cellular bodies were not specifically labeled. In the supracoronal region, for instance, both antibodies revealed the presence of an extensive basiepidermal nerve plexus spreading throughout the shell (Figure 3b-e). This plexus consisted of neurites with no obvious spatial orientation or organization (Figure $3 \mathrm{~d}, \mathrm{e}$ ). In addition, within the plexus, a limited number of local condensations were observed that were associated with the pedicellariae (Figure 3c). However, we found no neural condensations associated with the juvenile spines.

In the coronal region, our analyses also revealed the presence of various neurites. At the level of the definitive spines, neurites were found encircling the tubercles, running into the shafts, and extending between adjacent definitive spines (Figure $3 \mathrm{~b}, \mathrm{c}, \mathrm{f}, \mathrm{g}$ ). Transversal optical sections through the coronal region further highlighted the most prominent neural structures of $P$. lividus early juveniles: the five radial nerve cords and the circumoral nerve ring (Figure $3 f-i)$. As expected, the five radial nerve cords were in ambulacral positions, that is, aligned with the primary podia. They extended along the comminator muscles (the most prominent muscles of Aristotle's lantern Supporting Information Figure S4) to the pharynx, where they were joined together by the circumoral nerve ring (Figure $3 f-i)$. In addition, from transversal views, we were able to distinguish the neural structures associated with the primary and the secondary podia (Figure $3 f-i$ ) as well as nerve bundles emanating from the radial nerve cords and innervating the podia and the spines (Figure $3 f, g$ ). These views further validated the presence, in the coronal region, of the basiepidermal nerve plexus, although it was less conspicuous than in the supracoronal region (Figure $3 \mathrm{~h}$ ). Of note, 
we did not identify however any direct connection between the basiepidermal nerve plexus and the radial nerve cords or the circumoral nerve ring.

Lastly, in the infracoronal region, the basiepidermal nerve plexus was also clearly visible (Figure $3 \mathrm{j}, \mathrm{k})$. In contrast to what we observed in the supracoronal region, though, the plexus, which was located below the epidermis of the peristome, displayed a clear spatial organization, with radially arranged neurites directed towards the rim of the future mouth (Figure $3 \mathrm{j}, \mathrm{k}$ ).

\section{The radial nerve cords and the circumoral nerve ring}

As mentioned above, the most prominent features of the nervous system of 4-day-old early juveniles of $P$. lividus were the five radial nerve cords and the circumoral nerve ring (Figure 4a-g). The five radial nerve cords were anatomically undistinguishable from one another. They all originated at the midline of the animal, that is, at the base of the primary podia, and ran towards the oral side of the juvenile, lying between the comminator muscles and the epidermis (Figure 4a,b). At their oral end, they were joined together by five neurite tracts located below Aristotle's lantern and forming the circumoral nerve ring (Figure 4a-d). Each of these five neurite tracts was about 50 $\mu \mathrm{m}$ long and $5 \mu \mathrm{m}$ thick and, together, conferred a pentagonal shape to the circumoral nerve ring (Figure 4c,d).

Transversal sections through the radial nerve cords further revealed their organization into three layers (Figure 4e-g). In medial position, a large number of densely packed perikarya (or cellular bodies) formed a curved, bridge-shaped layer that was about $60 \mu \mathrm{m}$ wide and $15 \mu \mathrm{m}$ thick (Figure 4e-g). Close to the pharynx (i.e., on the inner side of the nerve cords), this layer of perikarya was restricted by a dense neuropile, likely constituted by neurites projected by the perikarya (Figure $4 \mathrm{e}-$ g). In cross-sections, this neuropile outlined a triangular-shaped layer that, at its largest point, was about $40 \mu \mathrm{m}$ wide and $20 \mu \mathrm{m}$ thick. Finally, close to the test (i.e., on the outer side of the nerve cords), the layer of perikarya was limited by a fluid-filled canal, the epineural canal (Figure 4e-g). This canal separated the perikarya from the endoskeleton and was particularly prominent in histological sections (Figure 4e). Of note, the acetylated $\alpha$-tubulin staining revealed, within the epineural canal, the presence of short, immunoreactive structures (Figure $4 \mathrm{f}$ ). However, given that acetylated $\alpha$-tubulin molecules are also present in cilia and that the synaptotagmin-B antibody did 
not label any structure within the lumen of the epineural canal (Figure 4g), we considered that this acetylated $\alpha$-tubulin staining marked short cilia from the perikarya.

Focusing on the circumoral nerve ring, we further identified an additional nerve ring located above it (i.e., between the circumoral nerve ring and Aristotle's lantern) (Figure 4h-l). This ring was composed of five pairs of neurite clusters that were positioned between the radial nerve cords and that were connected to one another as well as to the circumoral nerve ring by perceptible neurite tracts (Figure $4 \mathrm{i}-\mathrm{k}$ ). The tracts connecting the paired clusters to one another created yet another pentagonal structure, which was not directly superimposed on the circumoral nerve ring. Instead, the angles of the two pentagons were interconnected (Figure 4l). Based on the literature, the paired clusters we identified very likely correspond to the hyponeural ganglia previously reported by Cobb and Laverack in 1966. We thus decided to refer to the pentagonal-shaped structure formed by these clusters and their associated neurite tracts as the hyponeural nerve ring.

In the vicinity of the circumoral and the hyponeural nerve rings, we also observed a number of additional neurites associated with Aristotle's lantern and the epidermis of the peristome (Supporting Information Figure S6). As one example, we identified, associated with each of the hyponeural nerve ring angle, a nerve strand (Figure 4l), which further co-localized with the dental promoter muscles (Supporting Information Figure S6a-a").

\section{The neuroanatomy of the appendages}

The appendages of 4-day-old early juveniles of P. lividus further displayed complex innervation patterns (Figure 5). In both primary and secondary podia, we identified, for instance, a dense nerve plexus lining the entire epidermis of the stem, up to the papilla (Figure 5a-d). We observed that these plexuses were further separated from the muscle fibers of the stem of the podia by a thin layer of connective tissue (Figure $5 \mathrm{e}, \mathrm{f}$ ). In addition, embedded in these nerve plexuses, the podia displayed a small number of distinctive neural condensations that were distributed along and around the stem. Along the stem, we found three circumferential condensations running perpendicular to the stem (Figure 5a-d). The first one was positioned at the base of the podia. The second one surrounded the base of the papilla and was reminiscent of the basal nerve ring previously described in sea stars (Garm, 2017; Moore \& Thorndyke, 1993). Finally, the third one was located at the tip of the papilla 
and was connected to several nerve fibers that created a very characteristic cap shape over the tip of the papilla. Around the stem, a longitudinal condensation extended from the circumferential condensation located at the base of the papilla to that positioned at the base of the podia (Figure $5 \mathrm{~b}, \mathrm{c})$. At the base of the papilla, this longitudinal condensation was further connected to the circumferential condensation through a prominent neuropile that protruded laterally towards the surface of the epidermis (Figure 5b-d). Of note, we chose to term this neuropile the podia distal neuropile, and in all podia, whether primary or secondary, the podia distal neuropile and its related longitudinal condensation were always restricted to the oral face of the animal (Figure 5e-g).

The nervous system of the podia was also found to be innervated directly by the radial nerve cords. For the primary podia, which were aligned with the radial nerve cords, this innervation corresponded to two short terminal neurite bundles that extended from the tip of the radial nerve cords (Figure 5h). We named these neurite bundles the podia terminal nerves. The secondary podia, in contrast, which were positioned on either side of the radial nerve cords, were innervated by lateral neurite bundles that protruded from the sides of the radial nerve cords (Figure 5i,j). These neurites bundles, which we called the podia lateral nerves, emerged from the radial nerve cords as two bundles, which were more clearly observable with the synaptotagmin-B staining (Figure 5j).

At the level of the definitive spines, our analysis also revealed a complex innervation pattern (Figure $5 \mathrm{k}-\mathrm{t}$ ). Within the shaft, we observed six nerve bundles running along (Figure $5 \mathrm{k}, \mathrm{m}$ ) and located within the connective tissue between the trabeculae (Figure 5l). These nerve bundles, which we termed the spine nerves, further contacted several neurons dispersed within the epidermis along the shaft (Figure $5 \mathrm{k}, \mathrm{m}$ ). At the base of the definitive spines, the spine nerves further terminated within a dense circular neuropile, positioned perpendicular to the spine axis and restricted to the most proximal portion of the ring muscle (i.e., close to the shell) (Figure $5 \mathrm{k}, \mathrm{m}$ ). Within the tubercle, the perikarya at the origin of this neuropile were distinguishable in a more distal position (Figure $5 \mathrm{~m})$. Altogether, these perikarya and the circular neuropile have previously been called the nerve ring (Peters, 1985). Here, however, we chose to call this structure the definitive spine basal ganglion to avoid confusion with the circumoral or hyponeural nerve rings.

Importantly, we found that all definitive spine basal ganglia were connected to other definitive spine basal ganglia, to the basiepidermal nerve plexus, and to the radial nerve cords (Figure $5 k-t)$. 
Direct connections were observed, for instance, between each of the five aboral basal ganglia. These connections corresponded to long neurites spreading over the epidermis from one definitive spine quartet to another (Figure 5o). Equivalent direct connections were also identified between the oral basal ganglia of each definitive spine quartet (Figure 5q). For the medial basal ganglia of a given quartet, we found direct neurite connections with the aboral basal ganglion of the same quartet (Figure $\left.5 n^{\prime}, p\right)$. Moreover, we observed connections between the medial basal ganglia themselves that were established by both direct neurite bundles (Figure $5 r$ ) and the basiepidermal nerve plexus (Figure 5t). Likewise, the medial and oral basal ganglia of a given quartet were connected through the basiepidermal nerve plexus (Figure $5 \mathrm{t}$ ), which was further interacting with the aboral basal ganglia (Figure $5 \mathrm{k}, \mathrm{m}, \mathrm{o}$ ). Lastly, all definitive spine basal ganglia were also innervated directly by the radial nerve cords. For the aboral and medial basal ganglia, these innervations were supplied by lateral neurites extending directly from the two short terminal nerves innervating the primary podia (Figure $\left.5 n, n^{\prime}, r, s, t\right)$. In contrast, the oral basal ganglia were innervated by lateral nerves emerging from the sides of the radial nerve cords in a more proximal position, that is, in the vicinity of the podia lateral nerves (Figure $5 \mathrm{t}$ ).

Finally, we found that the pedicellariae were also associated with specific neural structures (Figure $5 \mathrm{u}-\mathrm{y}$ ). We identified three separate neurite tracts running between the adductor muscles (Figure $5 \mathrm{u}, \mathrm{v}$ ) and ascending perpendicular to them, towards the distal portion of the pedicellariae (Figure 5w-y). These neurite tracts are called the valve nerves (Peters \& Campbell, 1987). Moreover, the valve nerves were interconnected by three intervalve link nerves, which were positioned between the adductor and abductor muscles (Figure 5u-y). The intervalve link nerves were arranged in parallel to the muscles, thereby exhibiting the same characteristic triangular shape (Figure $5 u$ ). The perikarya associated with the valve and intervalve nerves were located in the pedicellaria epidermis, either at the distal tip of the valve nerves or below the intervalve link nerves (Figure 5x) (Peters \& Campbell, 1987). The nervous system of the pedicellariae was further connected to the basiepidermal nerve plexus through selective neurite bundles located at the level of the stem (Figure 5x,y). However, no connections were detected between the pedicellariae nervous system and the radial nerve cords. 


\section{Neuroanatomy of Patiria miniata and Parastichopus parvimensis juveniles}

In order to establish similarities and differences in nervous system organization among echinoderms, we subsequently investigated the neuromuscular anatomy of postmetamorphic individuals of the asteroid P. miniata and the holothuroid P. parvimensis (Figure 6) (for details on the external and muscular anatomies of $P$. miniata and $P$. parvimensis juveniles see Supporting Information Figure S7). In this context, the most obvious similarity observed, between the three species, was a general conservation of the organization of the nervous systems, with the presence of a basiepidermal nerve plexus spread all over the body, five radial nerve cords joined together by a circumoral nerve ring, and multiple peripheral nerves innervating the different appendages (Figure $6 a-m)$.

A more detailed analysis of each of these neural structures revealed a series of additional similarities. First, in P. miniata and P. parvimensis juveniles, like in P. lividus, we found that, although the basiepidermal nerve plexus spread throughout the body (Figure 6a-c), it was most evident on the aboral side (Figure 6d,e). Moreover, the asteroid and holothuroid basiepidermal nerve plexuses were also characterized by a meshwork of neurites with no specific spatial orientation or organization (Figure 6d,e). Second, in P. miniata and P. parvimensis, like in P. lividus, the five radial nerve cords were likewise distributed along the five ambulacra (Figure 6a,c), and the circumoral nerve ring was positioned on the oral side around the pharynx (Figure 6a,b). In P. miniata, the radial nerve cords further ran, like in $P$. lividus, underneath a set of muscles that resembled the $P$. lividus comminator muscles and that we named the radial muscles according to Sweet et al., 2019 (Figure 6f). Third, in P. miniata and P. parvimensis, like in P. lividus, several lateral nerves were further found protruding from the radial nerve cords and innervating the podia and the spines (Figure 6g,ik).

Similarities were also identified at the level of the neuroanatomy of the appendages, in particular at the level of the secondary podia and the spines (Figure $6 \mathrm{~g}-\mathrm{m}$ ). In all three species, we distinguished an almost identical nervous system architecture of the secondary podia (Figure 6g-i). In $P$. miniata and P. parvimensis, like in P. lividus, the epidermis of the secondary podia was entirely covered by a nerve plexus throughout the stem, up to the papilla (Figure $6 \mathrm{~h}, \mathrm{i}$ ). These plexuses were 
further similarly separated from the inner muscle fibers by a thin layer of connective tissue (Figure $6 g-i)$. In addition, along and around the stem, the asteroid and holothuroid secondary podia plexuses displayed at least one circumferential condensation at the base of the papilla (i.e., a basal nerve ring) and one longitudinal condensation that was also facing the oral side of the animal (Figure 6h,i). In the case of $P$. miniata, these two condensations were further connected through a prominent podia distal neuropile that was also facing the oral side of the animal, like in P. lividus (Figure 6h).

In regards to the spines, their associated neural structures could only be investigated in $P$. miniata juveniles, since $P$. parvimensis juveniles did not exhibit any spines. $P$. miniata juveniles possessed two types of spines: the juvenile spines on the aboral side (Supporting information Figure S7a") and the arm spines at the extremity of the interambulacra (Figure 6j). Compared to P. lividus definitive spines, $P$. miniata arm spines were flat and webbed together, at the two extremities of each interambulacrum, into a comb-like structure that usually included four to six spines (Figure 6j, Supporting Information Figure S7a). Interestingly, our analyses revealed that the innervation and connection patterns of the P. miniata arm spines and related webbed comb structures were very similar to those of the P. lividus definitive spines and related quartets (Figure 6j-m). Our acetylated $\alpha$-tubulin staining highlighted the presence, along the shaft of the arm spines, of long, thin nerve bundles (Figure 6j), reminiscent of the spine nerves in P. lividus. In P. miniata, however, these nerve bundles were not connected to a basal ganglion, like in P. lividus. Nonetheless, they were also innervated directly by the adjacent radial nerve cord through a protruding lateral nerve (Figure 6j,k). In addition, like in $P$. lividus, several connections were identified within the interambulacra of $P$. miniata juveniles, including connections between the two webbed combs of a given interambulacrum, between the webbed combs of distinct interambulacra, to the basiepidermal nerve plexus, and to the radial nerve cords (Figure $6 \mathrm{k}-\mathrm{m}$ ). For instance, we observed that each lateral neurite, emanating from a radial nerve cord and innervating the arm spines of a given webbed comb, also innervated the spine-free zone of the same interambulacrum through a distinct, more proximal, lateral protrusion (Figure $6 \mathrm{k}$ ). These protrusions, by interacting together, thus established a direct connection between the two webbed combs of a same interambulacrum (Figure 6j-1). In addition, connections between two webbed combs of two distinct interambulacra were established by nerve bundles located in the vicinity of the primary podia (Figure $6 \mathrm{~m}$ ). Finally, various 
conspicuous connections to the basiepidermal nerve plexus were further identified at the level of the lateral neurites innervating the arm spines and the spine-free zone of the interambulacra (Figure $61, \mathrm{~m})$.

In spite of these similarities, we also observed several species-specific neural features (Figure 6nq). In P. miniata juveniles, for instance, the stem of the primary podia was lined by an epidermal nerve plexus that was much denser than that of $P$. lividus primary podia (Figure 6j,n). Moreover, this plexus neither displayed any noticeable circumferential or longitudinal condensations, nor any podia distal neuropiles (Figure 6n). In contrast, the base of the asteroid primary podia was characterized by a large plexus-free zone, which corresponded to the location of the podia eye-spot (or optic cushion) (Petie et al., 2016).

In $P$. parvimensis juveniles, the basiepidermal nerve plexus spanning throughout the body was also particularly well developed, compared to that of the two other species (Figure 6b,c). However, given that metamorphosis progresses more slowly in holothuroids than in echinoids and asteroids (Morgan, 2008; Smiley, 1986), it could not be ruled out that, at the developmental stage investigated, a portion of the plexus we observed in P. parvimensis juveniles still included remnants of the nerves associated with the larval ciliary bands. Furthermore, in $P$. parvimensis juveniles, unlike in $P$. miniata and $P$. lividus, the five radial nerve cords were not anatomically equivalent. The ventral radial nerve cord, which innervated the secondary podium, was indeed much more developed than the other four radial nerve cords (Figure 6c). Even in terms of its inner organization, the ventral radial nerve cord was clearly distinct. It featured a large nerve bundle and a radial canal (Figure 6o), while the other four radial nerve cords were only composed of thin tracts of neurites surrounded by a layer of cellular bodies (Figure 6p). Finally, unlike the radial nerve cords of $P$. miniata and $P$. lividus, those of P. parvimensis did not terminate in the vicinity of the primary podia (Figure 6c). Instead, they terminated away from them. In holothuroids, the primary podia, which differentiate into buccal tentacles, develop at the level of the mouth (Díaz-Balzac, Abreu-Arbelo, \& García-Arrarás, 2010) and thus in the direct vicinity of the circumoral nerve ring (Figure 6c). In addition, in $P$. parvimensis, these podia were not innervated by terminal nerves protruding from the radial nerve cords, like in echinoid or asteroid juveniles. Instead, they were innervated by lateral nerves that arose directly from the circumoral nerve ring (Figure 6b,q). 


\section{Discussion}

The work reported here represents the first comprehensive description of the neural architecture of a postmetamorphic echinoid, relative to its endoskeleton and musculature. In addition, this work provides a comparison of the echinoid juvenile nervous system with those of a postmetamorphic asteroid and holothuroid. Altogether, we determined that the nervous system of an echinoid early juvenile is composed of three main structures: (a) a basiepidermal nerve plexus spread around the body, (b) a central structure composed of the five radial nerve cords and the circumoral and hyponeural nerve rings, and (c) several peripheral nerves innervating different muscles and appendages. We hypothesize that these three neural structures define two independent functional units: one acting locally, with on-site perception and read-out (using the basiepidermal nerve plexus) and one organized as a central unit, with integrative functions and motor and hormonal outputs (using the radial nerve cords plus the peripheral nerves). Our detailed analyses of whole mount preparations further enabled us to describe sophisticated innervation patterns at the level of several organs and appendages, including at the level of the podia and the spines. Comparisons of our results in echinoids with those we obtained in asteroids and holothuroids revealed a general conservation of the neural organization between the three species, with the presence of both three main neural components, which are likely to constitute the anatomical basis of two comparable, independent functional units, and some similar innervation patterns at the level of the appendages. It is thus likely that the last common ancestor of these three echinoderm classes already featured this conserved neural architecture. We hence argue that incorporating these conserved components of the echinoderm nervous system into broader comparisons of deuterostome nervous systems will be relevant and essential for a more complete understanding of deuterostome nervous system evolution. Future work should thus focus on in-depth comparisons between these components and those of chordate and hemichordate nervous systems.

\section{Neuroanatomy of Paracentrotus lividus early juveniles}

Previous analyses of the radial nerve cords and the basiepidermal nerve plexus of adult echinoids using electron microscopy have already defined the ultrastructure of these organs (Cobb, 1970; 
Märkel \& Röser, 1991; Weber \& Grosmann, 1977). Likewise, the innervations of the podia, of the definitive spines, and of Aristotle's lantern have previously been characterized in adult echinoids by electron microscopy and, to a lesser extent, immunohistochemistry (Agca et al., 2011; Cobb \& Laverack, 1966; Peters, 1985). Compared to this published body of work, our whole-mount immunohistochemistry approach on $P$. lividus early juveniles retrieved most of the known adult neural structures. A notable exception though is the absence of the nerve plexus associated with the viscera, which has yet previously been identified in several different echinoderm species (Cobb, 1969; Díaz-Balzac, Lázaro-Peña, Vázquez-Figueroa, Díaz-Balzac, \& García-Arrarás, 2016; Garcia-Arraras, Rojas-Soto, Jimenez, \& Diaz-Miranda, 2001; Mashanov et al., 2016). We believe that the absence of this neural plexus in our specimens is either due to some technical limitations of our experimental approach or related to the fact that early echinoid juveniles lack of a functional digestive tract (Burke, 1989; Gosselin \& Jangoux, 1998).

In echinoderms, the nervous system has traditionally been described as a superimposition of distinct layers of neural tissue referred to as the entoneural, ectoneural, and hyponeural subsystems (Hyman, 1955; Mashanov et al., 2016; Smith, 1965). The entoneural subsystem is found only in crinoids (Heinzeller \& Welsch, 2001), while the ectoneural and hyponeural subsystems are present in all echinoderm classes (Hyman, 1955; Mashanov et al., 2016; Smith, 1965). The hyponeural subsystem has previously been reported as particularly well developed in echinoderms with a conspicuous musculature, such as asteroids, ophiuroids, and holothuroids, while being reduced in echinoderms lacking an abundant musculature, such as echinoids and crinoids (Mashanov et al., 2016). The first description of the hyponeural subsystem of echinoids reported the presence, in Echinus esculentus, of ten ganglia located at the base of the radial nerve cords and devoted to the control of the muscles of Aristotle's lantern (Cobb \& Laverack, 1966). Two more recent descriptions, in Heliocidaris erythrogramma and Arbacia dufresnii, likewise reported the presence of, respectively, five groups of cells or ganglia in the echinoid hyponeural subsystem (Mashanov et al., 2016; Sly, Hazel, Popodi, \& Raff, 2002). Here, we identified five pairs of neurite clusters located aboral to the circumoral nerve ring, in a position that is consistent with the original description of the ten hyponeural ganglia by Cobb and Laverack in 1966. Our data thus support the hypothesis that the nervous system of adult 
echinoids includes a hyponeural subsystem that is however not only composed of ten ganglia but also of a nerve ring located aboral to the circumoral nerve ring.

In early juveniles of $P$. lividus, we identified three main neural structures: (a) a basiepidermal nerve plexus, (b) five radial nerve cords joined by a circumoral nerve ring, and (c) several peripheral nerves innervating different muscles and appendages. The basiepidermal nerve plexus appeared chiefly as a mesh-like network of neurites with no overt spatial orientation and organization. This planar arrangement, without any obvious hierarchy, is reminiscent of the nervous systems found in cnidarians and ctenophores. In these animals, the nerve nets are indeed loose networks of interconnected neurons, which receive sensory input and induce motor output locally (Arendt, Tosches, \& Marlow, 2016; Watanabe, Fujisawa, \& Holstein, 2009). In echinoids, the basiepidermal nerve plexus has also previously been proposed to be mostly sensory and to process this sensory information only locally (Cobb, 1968, 1970). For example, the neural condensations we found within the basiepidermal nerve plexus are connected to the pedicellariae, and the opening and closing movement of the jaws of these appendages have been established, in adults, to be completely independent of the radial nerve cords and to be exclusively controlled by the basiepidermal nerve plexus (Campbell, 1973; Campbell \& Laverack, 1968; von Uexküll, 1899). Similarly, the definitive spines, which we showed also to be connected to the basiepidermal nerve plexus, have been suggested to act as autonomous organs that can be controlled by local stimuli (Bullock, 1965; Kinosita, 1941; von Uexküll, 1900). In support of this view, when touching a specific point on the animal test, only the surrounding definitive spines move and converge towards the point of contact. Thus, the basiepidermal nerve plexus of echinoids may well correspond to an independent neural entity, functioning as a local unit with on-site perception and readout, without any input from the radial nerve cords.

In this work, we have further demonstrated that the radial nerve cords of $P$. lividus juveniles are composed of a large layer of perikarya and a dense neuropile defining a tubular structure located below the epidermis, and from which it is separated via the epineural canal. We have further shown that several terminal and lateral neurites project from the neuropile and innervate different organs and appendages, including Aristotle's lantern, podia, and definitive spines. From a purely anatomical point of view, this organization is reminiscent of the typical nervous system architecture of animals 
with a central and a peripheral nervous system, such as chordates (Holland, 2009). As previously proposed, the radial nerve cords of echinoids may thus act as an integrative center, with the peripheral nerves relaying information to and from this central unit (Burke, 2011; Haag, 2005; Mashanov et al., 2016; Smith, 1966). In this context, the nervous system of the podia might represent an important component of the echinoid peripheral nervous system. Consistent with this notion, the podia have been proposed to have important sensory functions, with sensory receptor cells and photosensory organs present at the tip of their papilla (Burke, 1980; Lesser, Carleton, Böttger, Barry, \& Walker, 2011) and with rhabdomeric opsins expressed at the tip and the base of their stem (Ullrich-Lüter, D'Aniello, \& Arnone, 2013; Ullrich-Lüter, Dupont, Arboleda, Hausen, \& Arnone, 2011). Interestingly, this expression co-localizes with the proximal and distal circumferential neural condensations that we observed in the podia of $P$. lividus juveniles. Local integration of the sensory information collected by the podia however seems unlikely, as we did not observe any direct connections between the neural structures of the podia and their muscle fibers. This statement agrees with previous work reporting the absence of axons crossing the connective tissue layer separating the podia epidermal nerve plexus and the muscle fibers (Florey \& Cahill, 1977). Yet, a local diffusion of acetylcholine through the connective tissue has been proposed to mediate contraction of the podia muscles (Florey \& Cahill, 1980). However, it remains unknown whether this regulation is controlled locally or centrally.

The definitive spines are also conspicuously innervated by lateral nerves issued from the radial nerve cords, thereby suggesting that these appendages are also an integral part of the echinoid peripheral nervous system. In support of this view, it is known that the definitive spines bear ciliated mechanosensory cells (Hyman, 1955) and initiate a coordinated wave movement when a shadow falls on the animal (Millott \& Takahashi, 1963), a reflex reaction that has been reported to be controlled by the radial nerve cords (Motokawa \& Fuchigami, 2015). As discussed above, however, there is also evidence that the definitive spines react to local stimuli relayed by the basiepidermal nerve plexus (Bullock, 1965; Kinosita, 1941; von Uexküll, 1900). The behavior of the definitive spines is thus likely to be controlled by both local and central neural stimuli, mediated respectively by the basiepidermal nerve plexus and the radial nerve cords. This notion, well established in the literature (Millott, 1966; Millott \& Takahashi, 1963; Peters, 1985), is in general agreement with our vision of the presence of 
two independent functional units in the echinoid nervous system: one acting locally, the basiepidermal nerve plexus, and one organized centrally, the radial nerve cords. It remains to be established, however, whether these two components of the echinoid nervous system are truly independent. Indeed, given their innervation pattern, the definitive spines might act as a hub between the basiepidermal nerve plexus and the radial nerve cords, allowing a certain degree of functional integration between the two components. Detailed neurophysiological analyses of the echinoid nervous system will be required to further address this issue.

\section{Comparison of echinoderm adult nervous systems}

Several similarities and differences between the nervous systems of adult echinoderms have previously been reported. One of the main differences is that the radial nerve cords of echinoids, holothuroids, and ophiuroids are subepidermal (Cobb, 1970; Märkel \& Röser, 1991), while those of asteroids and crinoids are basiepidermal (Heinzeller \& Welsch, 2001; Hyman, 1955; Smith, 1965). This means that, in echinoids, holothuroids, and ophiuroids, the radial nerve cords are internalized during their ontogeny and lie underneath the epidermis, while, in asteroids and crinoids, they remain superficially embedded in the epidermis. Another difference concerns the distribution of the hyponeural subsystem in the distinct echinoderm classes. In asteroids, ophiuroids, and crinoids, the hyponeural subsystem is present along both the circumoral nerve ring and the five radial nerve cords (Cobb \& Stubbs, 1982; Heinzeller \& Welsch, 1994; Hyman, 1955; Zueva, Khoury, Heinzeller, Mashanova, \& Mashanov, 2018). In contrast, in holothuroids and echinoids, the hyponeural subsystem is absent, respectively, along the circumoral nerve ring (Ehlers, 1997; Mashanov, Zueva, Heinzeller, \& Dolmatov, 2006) and the radial nerve cords (Mashanov et al., 2016). In echinoids, previous studies have shown that the hyponeural subsystem is composed of 10 ganglia located at the vicinity of the circumoral nerve ring (Cobb \& Laverack, 1966; Mashanov et al., 2016; Sly et al., 2002). Here, our work revealed that, in P. lividus juveniles, this subsystem is not restricted to 10 ganglia, but that it is established instead by the hyponeural nerve ring. In addition, while in asteroids, ophiuroids, and crinoids, this hyponeural nerve ring is directly superimposed on the circumoral nerve ring (Mashanov et al., 2016; Zueva et al., 2018), we found that the position of the hyponeural nerve ring in $P$. lividus juveniles is shifted relative to that of the radial nerve cords. 
In studying representatives of three different echinoderm classes, we were further able to identify some class-specific neural features. In the juveniles of the holothuroid P. parvimensis, for instance, we observed that, unlike in the juveniles of the echinoid P. lividus or the asteroid P. miniata, the five radial nerve cords are dissimilar. The anatomy of the ventral nerve cord of the holothuroid $P$. parvimensis is clearly different from that of the four other radial nerve cords. However, this difference might simply result from an asynchronous development of the radial nerve cords, as it has previously been proposed for the species Stichopus japonicus (Nakano et al., 2006). Furthermore, in P. parvimensis, the radial nerve cords do not terminate at the level of the primary podia (i.e., away from the circumoral nerve ring), and they do not protrude any peripheral nerves innervating these appendages. These characteristics, which are common in holothuroids (Mashanov et al., 2009), have already led some authors to question the homology of the radial nerve cords of holothuroids and the radial nerve cords of other echinoderms (Smiley, 1986).

In addition to specific differences, we have also identified during this work a number of conserved features between the nervous systems of echinoids, asteroids, and holothuroids. As previously reported for their adult counterparts, we found the juvenile nervous systems in the three species to be tripartite, with the presence of (i) a basiepidermal nerve plexus consisting of neurites with no obvious hierarchy, (ii) a centralized structure composed of five radial nerve cords and a circumoral nerve ring, and (iii) several peripheral nerves innervating the body musculature and the appendages. We further found that the neural architecture of some appendages is remarkably well conserved in all three species. At the level of the secondary podia, for example, we identified, in all three species, a comparable nerve plexus spanning the entire stem, that is separated from the inner muscle fibers by a thin layer of connective tissue, and that is characterized by the presence of circumferential and oral, longitudinal condensations. In addition, in all three species, the nervous system of the secondary podia is innervated by lateral neurites emanating from the radial nerve cords. These observations are consistent with previous reports on the neuroanatomy of the tube feet (i.e., secondary podia) in adult asteroids and holothuroids (Díaz-Balzac et al., 2010; Moore \& Thorndyke, 1993). In addition, in holothuroids, like in echinoids, the function of the tube foot (i.e., secondary podium) has been described to likely dependent upon the release of a signaling molecule, that is, histamine (Hoekstra, Moroz, \& Heyland, 2012). A similar innervation architecture has 
moreover recently been described in two members of a fourth echinoderm class, the ophiuroids Amphipholis kochii and Ophioderma brevispinum (Zueva et al., 2018). In these two species, the adult podia (i.e., secondary podia) are also characterized by a circumferential and an oral, longitudinal condensation, even though the former is positioned at the base of the podia. In addition, these neural structures are also innervated by peripheral nerves issued from the underlying radial nerve cords. Along the same lines, in the echinoid and asteroid interambulacra, we further identified similar innervation patterns, with connections to the basiepidermal nerve plexus, the radial nerve cords, and across and within the spine quartets or the spine webbed combs. Most notably, in both classes, we found the presence of spine nerves spreading all along the shaft of the spines and that are innervated by lateral nerves emanating from the radial nerve cords. In ophiuroids, spine nerves are also present, and they are similarly innervated by peripheral nerves protruding from the radial nerve cords (Wilkie, 2016; Zueva et al., 2018).

Despite distinctive, class-specific features, the three echinoderm classes we studied thus displayed nonetheless a remarkably conserved neural architecture. In addition, the data from ophiuroids suggest that at least some aspects of the neuroanatomy of these animals are also conserved with that of echinoids, asteroids, and holothuroids (Wilkie, 2016; Zueva et al., 2018). Together, these results hence suggest that the nervous system of the last common ancestor of these four echinoderm classes, that is, the eleutherozoan ancestor (Reich, Dunn, Akasaka, \& Wessel, 2015; Telford et al., 2014), already had a tripartite neural organization as well as a distinctive set of innervation patterns at the level of the appendages. We hypothesize that this tripartite neural organization of the eleutherozoan ancestor already established two functional units, one required for the interpretation of local sensory information (using the basiepidermal nerve plexus) and one capable of receiving peripheral sensory information and controlling behavioral outputs at the level of the whole animal (using the radial nerve cords, circumoral nerve ring and peripheral nerves). However, given the lack of information from crinoids, the most basal echinoderm class (Reich et al., 2015; Telford et al., 2014), it remains elusive when exactly this nervous system organization evolved, that is to say in the last common ancestor of eleutherozoans or in that of echinoderms. 


\section{Echinoderm adult nervous systems and deuterostome nervous systems evolution}

Due to the pentaradial symmetry of their bodies, adult echinoderms have so far been largely neglected as models for comparative, evolutionary analyses (Benito-Gutiérrez \& Arendt, 2009; Holland, 2015b). Yet, on the basis of the results we obtained here, we argue that the conserved features of the nervous system of adult echinoderms provide a robust framework for comparative analyses with animals from other phyla, in particular within the deuterostomes. The deuterostomes include the chordates, that is, vertebrates, tunicates, and cephalochordates, and the ambulacrarians, that is, hemichordates and echinoderms (Cannon et al., 2014; Satoh et al., 2014). In chordates, all nervous systems characterized to date are organized into a central structure, that is to say a neural tube giving rise to a brain (or cerebral vesicle) and a spinal cord, and a peripheral structure, which consists of a number of afferent and efferent nerves connecting sensory organs and effectors to the integrative central structure (Holland, 2009; Nielsen, 1999). In comparison, in hemichordates, the overall anatomy of the adult nervous system is very different. In these animals, the adult nervous system contains a basiepidermal nerve plexus, a couple of neural condensations, and several peripheral nerves (Kaul-Strehlow et al., 2015; Stach, Gruhl, \& Kaul-Strehlow, 2012). In enteropneust hemichordates, the best characterized of the hemichordate classes, the basiepidermal nerve plexus extends through the whole body, although it is more conspicuous in the proboscis. In addition, in these animals, there are two neural condensations, one located dorsally, which extends from the proboscis to the tail, and one positioned ventrally, which spreads exclusively through the trunk. These two condensations are further linked at the level of the pharynx in the collar by a circumferential condensation (Bullock, 1945; Kaul-Strehlow et al., 2015; Miyamoto, Nakajima, Wada, \& Saito, 2010). Despite this knowledge, our current understanding of hemichordate neurophysiology remains insufficient to establish whether this adult nervous system is capable of processing sensory information locally, globally, or both (Bullock, 1940; Knight-Jones, 1952). Furthermore, anatomical and molecular studies of the different components of the hemichordate adult nervous system have yielded conflicting hypotheses concerning their evolutionary relationships to the neural structures of chordates (Lowe et al., 2003; Nomaksteinsky et al., 2009; Pani et al., 2012). To date, establishing 
homologies between the hemichordate and chordate nervous systems thus remains a controversial issue (Holland, 2015b; Lowe et al., 2015).

Here, we propose that analyses of the adult nervous system of echinoderms have the potential to shed new light on the evolution of deuterostome nervous systems. Previous studies have suggested that the radial nerve cords of echinoids, holothuroids, and ophiuroids are homologous to the enteropneust hemichordate dorsal collar cord and the chordate neural tube (Burke, 2011; Formery et al., 2019; Haag, 2005; Morris, 2012). All these neural structures are indeed fluid-filled tubular canals that are composed of neurogenic ectoderm and that form through comparable tissue internalization processes (Bateson, 1884; Kaul \& Stach, 2010; Lowery \& Sive, 2004; Smiley, 1986; von Ubisch, 1913). However, equivalent subepidermal nerve cords have not yet been reported in any pterobranch hemichordates or asteroid and crinoid echinoderms, thereby complicating the interpretation of these neural structures in an evolutionary context. Along the same lines, the evolutionary relationship of the ambulacrarian basiepidermal nerve plexuses to the chordate nervous system remains elusive. In chordates, nerve plexuses presenting some degree of local, sensory autonomy have been described, for example, at the level of the vertebrate enteric nervous system or that of the cephalochordate pharyngeal nervous system (Kirtisinghe, 1940; Lacalli, Gilmour, \& Kelly, 1999; Nicol, 1952; Waxenbaum \& Varacallo, 2019). In both vertebrates and cephalochordates, though, these nerve plexuses seem to be connected to the central nervous system and might thus correspond to an integral part of the peripheral nervous system. In the enteropneust hemichordate Saccoglossus kowalevskii, recent molecular surveys have established that several orthologs of genes, involved in anterior-posterior patterning of the chordate neural tube, are expressed in similar patterns, but in the epidermis, which holds the basiepidermal nerve plexus (Aronowicz \& Lowe, 2006; Lowe et al., 2003; Pani et al., 2012). Epidermal expression of some of these anterior-posterior patterning genes have also been reported in the adult rudiment of an echinoid (Adachi et al., 2018; Tsuchimoto \& Yamaguchi, 2014) and in embryos and larvae of a cephalochordate (Schubert, Holland, Escriva, Holland, \& Laudet, 2004). However, the evolutionary interpretation of the conserved deployment of this genetic toolkit is still disputed and with it the question of the evolutionary history of deuterostome nervous systems (Formery et al., 2019; Holland, 2015b; Lowe et al., 2015). We hence propose that future work should focus on (i) the neurophysiological characterization of the adult 
echinoderm nervous system to assess the interplay of its components and (ii) the characterization of the molecular mechanisms underlying its patterning and development. Building on the present study, this additional work will allow detailed comparisons of individual components of the echinoderm, hemichordate, and chordate adult nervous systems, and thereby provide the key to resolving their evolutionary relationships.

\section{Acknowledgements}

The authors would like to thank Axel Wakefield for his support with the husbandry of P. lividus larvae and juveniles and Auston Rutledge for the husbandry of P. miniata and P. parvimensis larvae and juveniles. We are indebted to Guy Lhomond and François Lahaye for technical assistance. The authors would further like to thank David R. McClay and Robert D. Burke for their generous gift of the anti-MSPI30 and anti-synaptotagmin-B antibodies, respectively, as well as for fruitful discussions. We are also grateful for the support of the Institut de la Mer de Villefranche (IMEV) through the Service Moyen à la Mer, the Plateforme d'Imagerie par Microscopie (PIM), the service Mediterranean Culture Collection of Villefranche (MCCV) and the Service Aquariologie (SA), of the Centre de Ressources Biologiques (CRB), all of which are financed by EMBRC-France (ANR-10-INBS-02). The scanning electron microscopy of the Centre Commun de Microscopie Appliquée (CCMA) was funded by the Région Sud/Provence-Alpes Côte d'Azur, the Conseil Départemental des Alpes Maritimes and the GIS-IBiSA. This work was supported (LF, MS, CJL, and JCC) by the French Ministry of Research and Technology (2173/2015), the Japan Society for the Promotion of Science (PE18758), the André Picard Network (ANR-II-IDEX-0004-02) and the National Aeronautics and Space Administration Exobiology (NNXI3A168G).

\section{References}

Adachi, S., Niimi, I., Sakai, Y., Sato, F., Minokawa, T., Urata, M., Sehara-Fujisawa, A., Kobayashi, I., \& Yamaguchi, M. (2018). Anteroposterior molecular registries in ectoderm of the echinus rudiment. Developmental Dynamics, 247(12), 1297- 1307.

Agca, C., Elhajj, M. C., Klein, W. H., \& Venuti, J. M. (2011). Neurosensory and neuromuscular organization in tube feet of the sea urchin Strongylocentrotus purpuratus. Journal of Comparative Neurology, 519(17), 3566- 3579. 
Angerer, L. M., Yaguchi, S., Angerer, R. C., \& Burke, R. D. (2011). The evolution of nervous system patterning: insights from sea urchin development. Development, 138(17), 3613-3623.

Appeltans, W., Ahyong, S. T., Anderson, G., Angel, M. V., Artois, T., Bailly, N., Bamber, R., Barber, A., Bartsch, I., Berta, A., Błażewicz-Paszkowycz, M., Bock, P., Boxshall, G., Boyko, C. B., Brandão, S. N., Bray, R. A., Bruce, N. L., Cairns, S. D., Chan, T.-Y., ... Costello, M. J. (2012). The magnitude of global marine species diversity. Current Biology, 22(23), 2189- 2202.

Arendt, D., Tosches, M. A., \& Marlow, H. (2016). From nerve net to nerve ring, nerve cord and brain - evolution of the nervous system. Nature Reviews Neuroscience, 17(1), 61- 72 .

Arikawa, K., \& Williams, D. S. (1993). Acetylated alpha-tubulin in the connecting cilium of developing rat photoreceptors. Investigative Ophthalmology \& Visual Science, 34(6), 2145- 2149.

Arnone, M. I., Byrne, M., \& Martinez, P. (2015). Echinodermata. In A. Wanninger (Ed.), Evolutionary Developmental Biology of Invertebrates: Deuterostomia, 6, 1-58. Vienna: Springer.

Aronowicz, J., \& Lowe, C. J. (2006). Hox gene expression in the hemichordate Saccoglossus kowalevskii and the evolution of deuterostome nervous systems. Integrative and Comparative Biology, 46(6), 890-901.

Arshavskii, Yu. I., Kashin, S. M., Litvinova, N. M., Orlovskii, G. N., \& Fel'dman, A. G. (1976). Coordination of movements of the tube feet and arms of ophiurans during locomotion. Neurophysiology, 8(6), 476- 480.

Bateson, W. (1884). The early stages in the development of Balanoglossus (sp. incert.). Journal of Cell Science, s2-24(94), 208- 236.

Benito-Gutiérrez, E., \& Arendt, D. (2009). CNS evolution: new insight from the mud. Current Biology, 19(15), R640- R642.

Bisgrove, B. W., \& Raff, R. A. (1989). Evolutionary conservation of the larval serotonergic nervous system in a direct developing sea urchin. Development, Growth \& Differentiation, 31(4), 363-370.

Black, M. M., \& Keyser, P. (1987). Acetylation of alpha-tubulin in cultured neurons and the induction of alpha-tubulin acetylation in PC12 cells by treatment with nerve growth factor. Journal of Neuroscience, 7(6), 1833- 1842.

Bullock, T. H. (1940). The functional organization of the nervous system of Enteropneusta. Biological Bulletin, 79(1), 91- 113.

Bullock, T. H. (1945). The anatomical organization of the nervous system of Enteropneusta. Journal of Cell Science, s2-86(341), 55- 111.

Bullock, T. H. (1965). Comparative aspects of superficial conduction systems in echinoids 
and asteroids. American zoologist, 5, 545- 562.

Burke, R. D. (1980). Podial sensory receptors and the induction of metamorphosis in echinoids. Journal of Experimental Marine Biology and Ecology, 47(3), 223- 234.

Burke, R. D. (1989). Echinoderm metamorphosis: comparative aspects of the change in form. In M. Jangoux \& J. M. Lawrence (Éds.), Echinoderm Studies, 3, 81-108. Rotterdam: AA Balkema.

Burke, R. D. (2011). Deuterostome neuroanatomy and the body plan paradox. Evolution \& Development, 13(1), 110- 115.

Burke, R. D., Angerer, L. M., Elphick, M. R., Humphrey, G. W., Yaguchi, S., Kiyama, T., Liang, S., Mu, X., Agca, C., Klein, W. H., Brandhorst, B. P., Rowe, M., Wilson, K., Churcher, A. M., Taylor, J. S., Chen, N., Murray, G., Wang, D., Mellott, D., ... Thorndyke, M. C. (2006). A genomic view of the sea urchin nervous system. Developmental Biology, 300(1), 434- 460.

Campbell, A. C. (1973). Observations on the activity of echinoid pedicellariae. Marine Behaviour and Physiology, 2(1-4), 33- 61.

Campbell, A. C., \& Laverack, M. S. (1968). The responses of pedicellariae from Echinus esculentus (L.). Journal of Experimental Marine Biology and Ecology, 2(3), 191- 214.

Cannon, J. T., Kocot, K. M., Waits, D. S., Weese, D. A., Swalla, B. J., Santos, S. R., \& Halanych, K. M. (2014). Phylogenomic resolution of the hemichordate and echinoderm clade. Current Biology, 24(23), 2827- 2832.

Chia, F. S., \& Burke, R. D. (1978). Echinoderm metamorphosis: Fate of larval structures. In F.-S. Chia \& M. E. Rice (Eds.), Settlement and Metamorphosis of Marine Invertebrate Larvae, 219-234. New York: Elsevier.

Cobb, J. L. S. (1968). The fine structure of the pedicellariae of Echinus esculentus (L.). Journal of the Royal Microscopical Society, 88(2), 223- 233.

Cobb, J. L. S. (1969). The innervation of the oesophagus of the sea-urchin Heliocidaris erythrogramma. Zeitschrift Für Zellforschung Und Mikroskopische Anatomie, 98(3), 323- 332 .

Cobb, J. L. S. (1970). The significance of the radial nerve cords in asteroids and echinoids. Zeitschrift für Zellforschung und Mikroskopische Anatomie, 108(4), 457-474.

Cobb, J. L. S. (1987). Neurobiology of the Echinodermata. In M. A. Ali (Ed.), Nervous Systems in Invertebrates, 483-525. New York: Plenum Press.

Cobb, J. L. S., \& Laverack, M. S. (1966). The lantern of Echinus esculentus (L). II. Fine structure of hyponeural tissue and its connexions. Proceedings of the Royal Society of London. Series B. Biological Sciences, 164 (997), 641-650.

Cobb, J. L. S., \& Stubbs, T. R. (1982). The giant neurone system in ophiuroids. III. The detailed connections of the circumoral nerve ring. Cell and Tissue Research, 226(3), 675687. 
David, B., Mooi, R., \& Telford, M. (1995). The ontogenetic basis of Lovén's Rule clarifies homologies of the echinoid peristome. In R. Emson, A. B. Smith, \& A. C. Campbell (Eds.), Echinoderm Research 1995, 155-164. Rotterdam: AA Balkema.

Díaz-Balzac, C. A., Abreu-Arbelo, J. E., \& García-Arrarás, J. E. (2010). Neuroanatomy of the tube feet and tentacles in Holothuria glaberrima (Holothuroidea, Echinodermata). Zoomorphology, 129(1), 33- 43.

Díaz-Balzac, C. A., Lázaro-Peña, M. I., Vázquez-Figueroa, L. D., Díaz-Balzac, R. J., \& García-Arrarás, J. E. (2016). Holothurian nervous system diversity revealed by neuroanatomical analysis. PLOS ONE, 11(3), e0151129.

Dupont, S., Thorndyke, W., Thorndyke, M. C., \& Burke, R. D. (2009). Neural development of the brittlestar Amphiura filiformis. Development Genes and Evolution, 219(3), 159-166.

Ehlers, U. (1997). Ultrastructure of the statocysts in the apodous sea cucumber Leptosynapta inhaerens (Holothuroidea, Echinodermata). Acta Zoologica, 78(1), 61- 68.

Emlet, R. B. (2010). Morphological evolution of newly metamorphosed sea urchins - a phylogenetic and functional analysis. Integrative and Comparative Biology, 50(4), 571- 588.

Ezhova, O. V., Malakhov, V. V., \& Egorova, E. A. (2018). Axial complex and associated structures of the sea urchin Strongylocentrotus pallidus (Sars, G.O. 1871) (Echinodermata: Echinoidea). Journal of Morphology, 279(6), 792- 808.

Ferreira, A., \& Cáceres, A. (1989). The expression of acetylated microtubules during axonal and dendritic growth in cerebellar macroneurons which develop in vitro. Developmental Brain Research, 49(2), 205- 213.

Florey, E., \& Cahill, M. A. (1977). Ultrastructure of sea urchin tube feet. Cell and Tissue Research, 177(2), 195- 214.

Florey, E., \& Cahill, M. A. (1980). Cholinergic motor control of sea urchin tube feet: evidence for chemical transmission without synapses. Journal of Experimental Biology, 88(1), 281- 292.

Formery, L., Schubert, M., \& Croce, J. C. (2019). Ambulacrarians and the ancestry of deuterostome nervous systems. Results and Problems in Cell Differentiation, 68, 31- 59.

Gao, F., Thompson, J. R., Petsios, E., Erkenbrack, E., Moats, R. A., Bottjer, D. J., \& Davidson, E. H. (2015). Juvenile skeletogenesis in anciently diverged sea urchin clades. Developmental Biology, 400(1), 148- 158.

Garcia-Arraras, J. E., Rojas-Soto, M., Jimenez, L. B., \& Diaz-Miranda, L. (2001). The enteric nervous system of echinoderms: unexpected complexity revealed by neurochemical analysis. Journal of Experimental Biology, 204(5), 865- 873.

Garm, A. (2017). Sensory biology of starfish - with emphasis on recent discoveries in their visual ecology. Integrative and Comparative Biology, 57(5), 1082- 1092.

Gavilán, B., Perea-Atienza, E., \& Martínez, P. (2016). Xenacoelomorpha: a case of 
independent nervous system centralization? Philosophical Transactions of the Royal Society B: Biological Sciences, 371(1685), 20150039.

Ghyoot, M., De Ridder, C., \& Jangoux, M. (1987). Fine structure and presumed functions of the pedicellariae of Echinocardium cordatum (Echinodermata, Echinoida). Zoomorphology, 106(5), 279- 288.

Gosselin, P., \& Jangoux, M. (1998). From competent larva to exotrophic juvenile: a morphofunctional study of the perimetamorphic period of Paracentrotus lividus (Echinodermata, Echinoida). Zoomorphology, 118(1), 31- 43.

Haag, E. S. (2005). Echinoderm rudiments, rudimentary bilaterians, and the origin of the chordate CNS. Evolution \& Development, 7(4), 280- 281.

Haesaerts, D., Jangoux, M., \& Flammang, P. (2003). Study of the perimetamorphic period of the sea star Asterias rubens by scanning electron microscopy. In J.-P. Féral \& B. David (Eds.), Echinoderm Research 2001, 155-159. Lisse: Balkema.

Heinzeller, T., \& Welsch, U. (1994). Crinoidea. In F. W. Harrison \& F.-S. Chia (Eds.), Microscopic Anatomy of Invertebrates: Echinodermata, 14, 9-148. New York: Wiley-Liss.

Heinzeller, T., \& Welsch, U. (2001). The echinoderm nervous system and its phylogenetic interpretation. In G. Roth \& M. F. Wullimann (Eds.), Brain Evolution and Cognition, 41-75. New York: Wiley-Spektrum.

Hennebert, E., Santos, R., \& Flammang, P. (2012). Echinoderms don't suck: Evidence against the involvement of suction in tube foot attachment. Zoosymposia, 7(1), 25-32.

Hinman, V. F., \& Burke, R. D. (2018). Embryonic neurogenesis in echinoderms. Wiley Interdisciplinary Reviews: Developmental Biology, 7(4), e316.

Hirokawa, T., Komatsu, M., \& Nakajima, Y. (2008). Development of the nervous system in the brittle star Amphipholis kochii. Development Genes and Evolution, 218(1), 15- 21.

Hoekstra, L. A., Moroz, L. L., \& Heyland, A. (2012). Novel insights into the echinoderm nervous system from histaminergic and FMRFaminergic-like cells in the sea cucumber Leptosynapta clarki. PLOS ONE, 7(9), e44220.

Holland, L. Z. (2009). Chordate roots of the vertebrate nervous system: expanding the molecular toolkit. Nature Reviews Neuroscience, 10(10), 736- 746.

Holland, L. Z. (2015a). The origin and evolution of chordate nervous systems. Philosophical Transactions of the Royal Society B: Biological Sciences, 370(1684), 20150048.

Holland, L. Z. (2015b). Evolution of basal deuterostome nervous systems. Journal of Experimental Biology, 218(4), 637- 645.

Hyman, L. H. (1955). The Invertebrates: Echinodermata (Vol. 4). New York: McGrawHill.

Jensen, M. (1985). Functional morphology of test, lantern and tube feet ampullae system in flexible and rigid sea urchins (Echinoidea). In B. F. Keegan \& B. D. S. O’Connor (Eds.), 
Echinodermata, 1984, 281-288. Rotterdam: AA Balkema.

Katow, H., Elia, L., \& Byrne, M. (2009). Development of nervous systems to metamorphosis in feeding and non-feeding echinoid larvae, the transition from bilateral to radial symmetry. Development Genes and Evolution, 219(2), 67- 77.

Kaul, S., \& Stach, T. (2010). Ontogeny of the collar cord: neurulation in the hemichordate Saccoglossus kowalevskii. Journal of Morphology, 271(10), 1240- 1259.

Kaul-Strehlow, S., Urata, M., Minokawa, T., Stach, T., \& Wanninger, A. (2015). Neurogenesis in directly and indirectly developing enteropneusts: of nets and cords. Organisms Diversity \& Evolution, 15(2), 405- 422.

Ke, M.-T., Fujimoto, S., \& Imai, T. (2013). SeeDB: a simple and morphology-preserving optical clearing agent for neuronal circuit reconstruction. Nature Neuroscience, 16(8), 1154- 1161.

Kinosita, H. (1941). Conduction of impulse in superficial nervous system of sea-urchin. Japanese Journal of Zoology, 9, 221- 232.

Kirtisinghe, P. (1940). Memoirs: the myenteric nerve-plexus in some lower chordates. Journal of Cell Science, s2-81(324), 521- 539.

Knight-Jones, E. W. (1952). On the nervous system of Saccoglossus cambrensis (Enteropneusta). Philosophical Transactions of the Royal Society B: Biological Sciences, 236(634), 315-354.

Lacalli, T. C., Gilmour, T. H. J., \& Kelly, S. J. (1999). The oral nerve plexus in amphioxus larvae: function, cell types and phylogenetic significance. Proceedings of the Royal Society B: Biological Sciences, 266(1427), 1461.

Lepage, T., \& Gache, C. (1989). Purification and characterization of the sea urchin embryo hatching enzyme. Journal of Biological Chemistry, 264(9), 4787- 4793.

Lesser, M. P., Carleton, K. L., Böttger, S. A., Barry, T. M., \& Walker, C. W. (2011). Sea urchin tube feet are photosensory organs that express a rhabdomeric-like opsin and PAX6. Proceedings of the Royal Society B: Biological Sciences, 278(1723), 3371-3379.

Lowe, C. J., Clarke, D. N., Medeiros, D. M., Rokhsar, D. S., \& Gerhart, J. (2015). The deuterostome context of chordate origins. Nature, 520(7548), 456- 465.

Lowe, C. J., \& Wray, G. A. (2000). Rearing larvae of sea urchins and sea stars for developmental studies. In J. M. Walker, R. S. Tuan, \& C. W. Lo (Eds.), Developmental Biology Protocols, 1, 9-15. Totowa: Humana Press.

Lowe, C. J., Wu, M., Salic, A., Evans, L., Lander, E., Stange-Thomann, N., Gruber, C. E., Gerhart, J., \& Kirschner, M. (2003). Anteroposterior patterning in hemichordates and the origins of the chordate nervous system. Cell, 113(7), 853- 865.

Lowery, L. A., \& Sive, H. (2004). Strategies of vertebrate neurulation and a re-evaluation of teleost neural tube formation. Mechanisms of Development, 121(10), 1189- 1197. 
Märkel, K., \& Röser, U. (1991). Ultrastructure and organization of the epineural canal and the nerve cord in sea urchins (Echinodermata, Echinoida). Zoomorphology, 110(5), 267- 279.

Mashanov, V. S., Zueva, O. R., \& Garcia-Arraras, J. E. (2015). Heterogeneous generation of new cells in the adult echinoderm nervous system. Frontiers in Neuroanatomy, 9, 123.

Mashanov, V. S., Zueva, O. R., Heinzeller, T., Aschauer, B., Naumann, W. W., Grondona, J. M., Cifuentes, M., \& Garcia-Arraras, J. E. (2009). The central nervous system of sea cucumbers (Echinodermata: Holothuroidea) shows positive immunostaining for a chordate glial secretion. Frontiers in Zoology, 6(1), 11.

Mashanov, V. S., Zueva, O. R., Heinzeller, T., \& Dolmatov, I. Y. (2006). Ultrastructure of the circumoral nerve ring and the radial nerve cords in holothurians (Echinodermata). Zoomorphology, 125(1), 27- 38.

Mashanov, V. S., Zueva, O. R., Rubilar, T., Epherra, L., \& Garcia-Arraras, J. E. (2016). Echinodermata. In A. Schmidt-Rhaesa, S. Harzsch, \& G. Purschke (Eds.), Structure and Evolution of Invertebrate Nervous Systems, 665-688. Oxford: Oxford University Press.

Matsuzaka, Y., Sato, E., Kano, T., Aonuma, H., \& Ishiguro, A. (2017). Non-centralized and functionally localized nervous system of ophiuroids: evidence from topical anesthetic experiments. Biology Open, 6(4), 425- 438.

McClay, D. R., Cannon, G. W., Wessel, G. M., Fink, R. D., \& Marchase, R. B. (1983). Patterns of antigenic expression in early sea urchin development. In W. R. Jeffrey \& R. A. Raff (Eds.), Time, Space, and Pattern in Embryonic Development, 69, 157-169. New York: Liss.

Mercurio, S., Gattoni, G., Messinetti, S., Sugni, M., \& Pennati, R. (2019). Nervous system characterization during the development of a basal echinoderm, the feather star Antedon mediterranea. Journal of Comparative Neurology, 527(6), 1127- 1139.

Millott, N. (1966). Coordination of spine movements in echinoids. In R. A. Boolootian (Ed.), Physiology of Echinodermata, 465-487. New York: Interscience.

Millott, N., \& Takahashi, K. (1963). The shadow reaction of Diadema antillarum Philippi. IV. Spine movements and their implications. Philosophical Transactions of the Royal Society of London. Series B, Biological Sciences, 246(736), 437- 469.

Miyamoto, N., Nakajima, Y., Wada, H., \& Saito, Y. (2010). Development of the nervous system in the acorn worm Balanoglossus simodensis: insights into nervous system evolution. Evolution \& Development, 12(4), 416- 424.

Mooi, R., \& David, B. (2008). Radial symmetry, the anterior/posterior axis, and echinoderm Hox genes. Annual Review of Ecology, Evolution, and Systematics, 39(1), 43- 62.

Mooi, R., David, B., \& Wray, G. A. (2005). Arrays in rays: terminal addition in echinoderms and its correlation with gene expression. Evolution \& Development, 7(6), 542- 555 .

Moore, S. J., \& Thorndyke, M. C. (1993). Immunocytochemical mapping of the novel echinoderm neuropeptide SALMFamide 1 (S1) in the starfish Asterias rubens. Cell and 
Tissue Research, 274(3), 605- 618.

Morgan, A. (2008). Metamorphosis in larvae of the temperate sea cucumber Australositchopus mollis. Invertebrate Reproduction \& Development, 51(3), 127- 143.

Morris, V. B. (2009). On the sites of secondary podia formation in a juvenile echinoid: growth of the body types in echinoderms. Development Genes and Evolution, 219(11), 597- 608 .

Morris, V. B. (2012). Early development of coelomic structures in an echinoderm larva and a similarity with coelomic structures in a chordate embryo. Development Genes and Evolution, 222(6), 313-323.

Motokawa, T., \& Fuchigami, Y. (2015). Coordination between catch connective tissue and muscles through nerves in the spine joint of the sea urchin Diadema setosum. Journal of Experimental Biology, 218(5), 703- 710.

Nakajima, Y., Kaneko, H., Murray, G., \& Burke, R. D. (2004). Divergent patterns of neural development in larval echinoids and asteroids. Evolution \& Development, 6(2), 95- 104.

Nakano, H., Murabe, N., Amemiya, S., \& Nakajima, Y. (2006). Nervous system development of the sea cucumber Stichopus japonicus. Developmental Biology, 292(1), 205- 212.

Nichols, D. (1972). The water-vascular system in living and fossil echinoderms. Palaeontology, 15(4), 519- 538.

Nicol, J. C. (1952). Autonomic nervous systems in lower chordates. Biological Reviews, 27(1), 1- 48 .

Nielsen, C. (1999). Origin of the chordate central nervous system - and the origin of chordates. Development Genes and Evolution, 209(3), 198- 205.

Nomaksteinsky, M., Röttinger, E., Dufour, H. D., Chettouh, Z., Lowe, C. J., Martindale, M. Q., \& Brunet, J.-F. (2009). Centralization of the deuterostome nervous system predates chordates. Current Biology, 19(15), 1264- 1269.

Pani, A. M., Mullarkey, E. E., Aronowicz, J., Assimacopoulos, S., Grove, E. A., \& Lowe, C. J. (2012). Ancient deuterostome origins of vertebrate brain signalling centres. Nature, 483(7389), 289- 294.

Peters, B. H. (1985). The innervation of spines in the sea-urchin Echinus esculentus L. Cell and Tissue Research, 239(1), 219- 228.

Peters, B. H., \& Campbell, A. C. (1987). Morphology of the nervous and muscular systems in the heads of pedicellariae from the sea urchin Echinus esculentus L. Journal of Morphology, 193(1), 35- 51.

Peters, E. C., Price, K. L., \& Horowitz, D. J. B. (2005). Histological preparation of invertebrates for evaluating contaminant effects. In G. K. Ostrander (Ed.), Techniques in Aquatic Toxicology, 2, 673-706. Boca Raton: CRC Press - Taylor \& Francis Group. 
Petie, R., Garm, A., \& Hall, M. R. (2016). Crown-of-thorns starfish have true image forming vision. Frontiers in Zoology, 13(1), 41.

Reich, A., Dunn, C., Akasaka, K., \& Wessel, G. (2015). Phylogenomic analyses of Echinodermata support the sister groups of Asterozoa and Echinozoa. PLOS ONE, 10(3), e0119627.

Richter, S., Loesel, R., Purschke, G., Schmidt-Rhaesa, A., Scholtz, G., Stach, T., Vogt, L., Wanninger, A., Brenneis, G., Döring, C., Faller, S., Fritsch, M., Grobe, P., Heuer, C. M., Kaul, S., Møller, O. S., Müller, C. H., Rieger, V., Rothe, B. H., ... Harzsch, S. (2010). Invertebrate neurophylogeny: suggested terms and definitions for a neuroanatomical glossary. Frontiers in Zoology, 7(1), 29.

Santos, R., Gorb, S., Jamar, V., \& Flammang, P. (2005). Adhesion of echinoderm tube feet to rough surfaces. Journal of Experimental Biology, 208(13), 2555-2567.

Satoh, N., Rokhsar, D., \& Nishikawa, T. (2014). Chordate evolution and the three-phylum system. Proceedings of the Royal Society B: Biological Sciences, 281(1794), 20141729.

Schneider, C. A., Rasband, W. S., \& Eliceiri, K. W. (2012). NIH Image to ImageJ: 25 years of image analysis. Nature Methods, 9, 671- 675.

Schubert, M., Holland, N. D., Escriva, H., Holland, L. Z., \& Laudet, V. (2004). Retinoic acid influences anteroposterior positioning of epidermal sensory neurons and their gene expression in a developing chordate (amphioxus). Proceedings of the National Academy of Sciences, 101(28), 10320- 10325.

Sly, B. J., Hazel, J. C., Popodi, E. M., \& Raff, R. A. (2002). Patterns of gene expression in the developing adult sea urchin central nervous system reveal multiple domains and deepseated neural pentamery. Evolution \& Development, 4(3), 189- 204.

Smiley, S. (1986). Metamorphosis of Stichopus californicus (Echinodermata: Holothuroidea) and its phylogenetic implications. Biological Bulletin, 171(3), 611- 631.

Smith, A. B. (2008). Deuterostomes in a twist: the origins of a radical new body plan. Evolution \& Development, 10(4), 493- 503.

Smith, J. E. (1937). The structure and function of the tube feet in certain echinoderms. Journal of the Marine Biological Association of the United Kingdom, 22(1), 345-357.

Smith, J. E. (1965). Echinodermata. In T. H. Bullock \& G. A. Horridge (Eds.), Structure and Function in the Nervous Systems of Invertebrates, II, 1519-1558. San Francisco and London: Freeman and Company.

Smith, J. E. (1966). The form and functions of the nervous system. In R. A. Boolootián (Ed.), Physiology of Echinodermata, 503- 511. New York: Interscience.

Stach, T., Gruhl, A., \& Kaul-Strehlow, S. (2012). The central and peripheral nervous system of Cephalodiscus gracilis (Pterobranchia, Deuterostomia). Zoomorphology, 131, 11- 24 .

Stauber, M. (1993). The lantern of Aristotle: organization of its coelom and origin of its 
muscles (Echinodermata, Echinoida). Zoomorphology, 113(2), 137- 151.

Sumner-Rooney, L., Rahman, I. A., Sigwart, J. D., \& Ullrich-Lüter, E. (2018). Wholebody photoreceptor networks are independent of 'lenses' in brittle stars. Proceedings of the Royal Society B: Biological Sciences, 285(1871), 20172590.

Sweet, H. C., Doolin, M. C., Yanowiak, C. N., Coots, A. D., Freyn, A. W., Armstrong, J. M., \& Spiecker, B. J. (2019). Abbreviated development of the brooding brittle star Ophioplocus esmarki. The Biological Bulletin, 236(2), 75- 87.

Telford, M. J., Lowe, C. J., Cameron, C. B., Ortega-Martinez, O., Aronowicz, J., Oliveri, P., \& Copley, R. R. (2014). Phylogenomic analysis of echinoderm class relationships supports Asterozoa. Proceedings of the Royal Society B: Biological Sciences, 281(1786), 20140479.

Tinoco, A. B., Semmens, D. C., Patching, E. C., Gunner, E. F., Egertová, M., \& Elphick, M. R. (2018). Characterization of NGFFYamide signaling in starfish reveals roles in regulation of feeding behavior and locomotory systems. Frontiers in Endocrinology, 9, 507.

Tsuchimoto, J., \& Yamaguchi, M. (2014). Hox expression in the direct-type developing sand dollar Peronella japonica. Developmental Dynamics, 243(8), 1020-1029.

Ullrich-Lüter, E. M., D’Aniello, S., \& Arnone, M. I. (2013). C-opsin expressing photoreceptors in echinoderms. Integrative and Comparative Biology, 53(1), 27- 38.

Ullrich-Lüter, E. M., Dupont, S., Arboleda, E., Hausen, H., \& Arnone, M. I. (2011). Unique system of photoreceptors in sea urchin tube feet. Proceedings of the National Academy of Sciences, 108(20), 8367-8372.

von Ubisch, L. (1913). Die Entwicklung von Strongylocentrotus lividus. Zeitschrift für Wissenschaftliche Zoologie, 106, 409-488.

von Uexküll, J. (1899). Die Physiologie der Pedicellarien. Zeitschrift Für Biologie, 37, $358-400$.

von Uexküll, J. (1900). Die Physiologie des Seeigelstachels. Zeitschrift Für Biologie, 39, 73-112.

Watanabe, H., Fujisawa, T., \& Holstein, T. W. (2009). Cnidarians and the evolutionary origin of the nervous system. Development, Growth \& Differentiation, 51(3), 167- 183.

Waxenbaum, J. A., \& Varacallo, M. (2019). Anatomy, Autonomic Nervous System. Treasure Island: StatPearls Publishing.

Weber, W., \& Grosmann, M. (1977). Ultrastructure of the basiepithelial nerve plexus of the sea urchin, Centrostephanus longispinus. Cell and Tissue Research, 175(4), 551- 562.

Wilkie, I. C. (1984). Variable tensility in echinoderm collagenous tissues: a review. Marine Behaviour and Physiology, 11(1), 1- 34.

Wilkie, I. C. (2002). Is muscle involved in the mechanical adaptability of echinoderm mutable collagenous tissue? The Journal of experimental biology, 205, 159- 165. 
Wilkie, I. C. (2016). Functional morphology of the arm spine joint and adjacent structures of the brittlestar Ophiocomina nigra (Echinodermata: Ophiuroidea). PLoS ONE, 11(12), 136.

Wray, G. A., \& Raff, R. A. (1991). The evolution of developmental strategy in marine invertebrates. Trends in Ecology \& Evolution, 6(2), 45- 50.

Zieger, E., Candiani, S., Garbarino, G., Croce, J. C., \& Schubert, M. (2018). Roles of retinoic acid signaling in shaping the neuronal architecture of the developing amphioxus nervous system. Molecular Neurobiology, 55(6), 5210- 5229.

Ziegler, A., Faber, C., \& Bartolomaeus, T. (2009). Comparative morphology of the axial complex and interdependence of internal organ systems in sea urchins (Echinodermata: Echinoidea). Frontiers in Zoology, 6(1), 10.

Ziegler, A., Schröder, L., Ogurreck, M., Faber, C., \& Stach, T. (2012). Evolution of a novel muscle design in sea urchins (Echinodermata: Echinoidea). PLOS ONE, 7(5), e37520.

Zueva, O., Khoury, M., Heinzeller, T., Mashanova, D., \& Mashanov, V. (2018). The complex simplicity of the brittle star nervous system. Frontiers in Zoology, 15(1), 1.

\section{Figure captions}

\section{Figure 1: External anatomy of Paracentrotus lividus early juveniles}

(a-c) Scanning electron microscopy images of whole mount specimens in (a) lateral view with oral side down, (b) aboral view and (c) oral view. In (a), the colored dotted lines delineate, along the oral-aboral axis of the animal (from bottom to top), the infracoronal, the coronal and the supracoronal regions. In (c), the orange dotted lines highlight the position of the five ambulacra, while the green dotted line marks an interambulacrum. a, aboral definitive spine; Amb, ambulacrum; $\mathrm{BP}$, buccal podium; CR, coronal region; DS, definitive spine; ICR, infracoronal region; Inter, interambulacrum; JS, juvenile spine; $\mathrm{m}$, medial definitive spine; $\mathrm{o}$, oral definitive spine; Ped, pedicellaria; Peri, peristome; PP, primary podium; SCR, supracoronal region; SP, secondary podium.

\section{Figure 2: Skeletal and muscular anatomy of Paracentrotus lividus early juveniles}


(a) Schematic representation indicating the width and position of the confocal stacks displayed in (b-

e) along the oral-aboral axis of the juvenile. (b-e) Maximum intensity projections of confocal z-stacks for specimens co-labeled, in (b, c), for MSP-130 (endoskeleton; red) and DNA (nuclei; blue) or colabeled, in (d, e), for F-actin (muscles; cyan) and DNA (nuclei; blue). Specimens are in (b, d) aboral and (c, e) oral views. In (b, d), the white dotted lines demarcate pedicellariae. In (c), the dotted lines delineate the 10 buccal plates surrounding the pharyngeal area. In (e), the red dotted line outlines Aristotle's lantern, and the white dotted line delineates the pharyngeal area. AxComp, axial complex; DS, definitive spine; JS, juvenile spine; Mes, mesentery; Ped, pedicellaria; Phx, pharynx; PP, primary podium; SP, secondary podium.

\section{Figure 3: General overview of the neuroanatomy of Paracentrotus lividus early}

\section{juveniles}

(a) Schematic representation displaying the width and position of the confocal stacks shown in (b, c, $\mathrm{f}-\mathrm{k}$ ) along the oral-aboral axis of the juvenile. (b-k) Maximum intensity projections of confocal zstacks for specimens co-labeled, in (b, d, f, h, j), for acetylated $\alpha$-tubulin (AcTub) (neurons and cilia; yellow), F-actin (muscles; cyan), and DNA (nuclei; blue) or labeled, in (c, e, g, i, k), for synaptotagmin-B (SytB) (neurons; green), exceptionally counterstained in (e) for DNA (nuclei; blue). $(b, c, f-k)$ Whole mount specimens in $(b, c)$ aboral, $(f-i)$ transversal, and $(j, k)$ oral views. $(d, e)$ Close-ups of the basiepidermal nerve plexus in the supracoronal region. $\ln (b, c, f, g)$, the thick white arrows point to neurites extending between adjacent definitive spines. In $(c)$, the dotted lines outline the position of pedicellariae. In $(f-i)$, the red asterisks mark the radial nerve cords. In $(f)$, the yellow asterisks indicate the comminator muscles. In ( $f, g)$, the green arrows highlight nerve bundles emanating from the radial nerve cords and innervating the secondary podia, and the red arrows highlight nerve bundles issued from the radial nerve cords and innervating the definitive spines. In (h), the orange arrowheads point to the basiepidermal nerve plexus in the coronal region. In $(j, k)$, the white arrowheads indicate radially arranged neurites of the basiepidermal nerve plexus in the infracoronal region. CNR, circumoral nerve ring; DS, definitive spine; DS shaft, definitive spine shaft; 
DSTub, definitive spine tubercle; JS, juvenile spine; Phx, pharynx; PP, primary podium; SP, secondary podium.

\section{Figure 4: The radial nerve cords, circumoral nerve ring and hyponeural nerve}

\section{ring of Paracentrotus lividus juveniles}

(a, c, f, g, h-l) Maximum intensity projections of confocal z-stacks for specimens co-labeled, in (a, c,

g), for synaptotagmin-B (SytB) (neurons; green), F-actin (muscles; cyan), and DNA (nuclei; blue), colabeled, in (f), for acetylated $\alpha$-tubulin (AcTub) (neurons and cilia; yellow), F-actin (muscles; cyan), and DNA (nuclei; blue) or labeled, in (h-l), for synaptotagmin-B (SytB). (b, d) Computed 3D reconstructions of specimens co-labeled for synaptotagmin-B (SytB) (neurons; green) and F-actin (muscles; cyan). (e) Histological section through a radial nerve cord. (a, b) Lateral and (c, d) oral views illustrating the position of the radial nerve cords and the circumoral nerve ring relative to the comminator muscles, the primary podia, and Aristotle's lantern. (e-g) Transversal sections through a radial nerve cord. In $(f, g)$, the dotted line indicates the position of the epineural canal. In $(f)$, the arrows mark the presence of cilia within the epineural canal. (h-k) Close-ups of the hyponeural nerve ring. In (h-k), the four projections are from the same confocal z-stack and were color-coded to demonstrate the relative position of different structures along the oral-aboral axis of the animal. (l) Composite image illustrating the relative position, oral (blue) versus aboral (yellow), of the hyponeural nerve ring compared to the radial nerve cords and the circumoral nerve ring. In (1), the asterisks indicate straight nerve bundles positioned at each angle of the hyponeural nerve ring. CNR, circumoral nerve ring; ComM, comminator muscle; EndoSk, endoskeleton; EpiC, epineural canal; HypClu, hyponeural cluster; HypNR, hyponeural nerve ring; Nrp, neuropile; Phx, pharynx; Pk, perikarya; PP, primary podium; RNC, radial nerve cord; SP, secondary podium.

\section{Figure 5: Neuroanatomy of the appendages of Paracentrotus lividus juveniles}

(a-c, e-m, o-x) Maximum intensity projections of confocal z-stacks for specimens co-labeled, in (a-c, e, f), for synaptotagmin-B (SytB) (neurons; green), F-actin (muscles; cyan), and DNA (nuclei; blue), co-labeled, in ( $\mathrm{g}-\mathrm{i}, \mathrm{k}, \mathrm{v}, \mathrm{w}$ ), for acetylated $\alpha$-tubulin (AcTub) (neurons and cilia; yellow), F-actin 
(muscles; cyan), and DNA (nuclei; blue), co-labeled, in $(j, m, x)$, for synaptotagmin-B (SytB) (neurons; green) and DNA (nuclei; blue) or co-labeled, in (l, o-u), for acetylated $\alpha$-tubulin (AcTub) (neurons and cilia; yellow) and DNA (nuclei; blue). (d, n, n', y) Computed 3D reconstructions of specimens co-labeled for synaptotagmin-B (neurons; green) and F-actin (muscles; cyan). (a-j) Neuroanatomy of primary and secondary podia. (a, b, d) Primary podia and (c) secondary podia in lateral views. (e-j) Primary and secondary podia in transversal views. In (a-d), the yellow arrowheads indicate the three circumferential condensations along the stem. In $(b, c)$, the white arrows highlight the oral longitudinal condensations. In (d), the red dotted line marks the position of the skeletal disk. In (h), the red arrows point to the podia terminal nerves that innervate the nervous system of a primary podium. In $(i, j)$, the red arrowheads show the podia lateral nerves emanating from the radial nerve cord and supplying the nervous system of the secondary podia. (k-t) Neuroanatomy of the definitive spines. $(k-l)$ Definitive spines in $(k, m)$ lateral and (l) transversal views. $(k, m)$ Closeups of the tubercle and the proximal part of the shaft. (l) Cross-section through the shaft. $\ln (k, m)$, the white arrows indicate dispersed neurons in the spine shaft, and the white arrowheads highlight connections between the definitive spine basal ganglia and the basiepidermal nerve plexus. In $(\mathrm{m})$, the dotted line outlines the cluster of perikarya present in the tubercle. (n-t) Close-ups of the distinct neural connections observed at the level of the definitive spine basal ganglia. In ( $n, r)$, the red arrows mark the two short terminal nerves issued from a radial nerve cord and innervating a primary podium. In $\left(n, n^{\prime}, r, t\right)$, the cyan arrowheads highlight lateral neurites issued from the podia terminal nerves and connecting the medial definitive spine ganglia. In (n, n', s), the magenta arrowheads indicate lateral neurites protruding from the podia terminal nerves and innervating an aboral definitive spine basal ganglion. In ( $\left.n^{\prime}, p\right)$, the yellow arrowheads mark neural bundles connecting an aboral definitive spine basal ganglion and its two adjacent medial definitive spine basal ganglia. In (o), the white arrow highlights a connection between two aboral definitive spine ganglia, and the white arrowheads indicate connections between an aboral definitive spine basal ganglion and the basiepidermal nerve plexus. In $(q)$, the cyan arrows mark connections between oral definitive spine basal ganglia. In (r), the orange arrowheads point to nerve bundles establishing connections between adjacent medial definitive spine basal ganglia. In $(t)$, the white asterisks highlight the basiepidermal nerve plexus connecting two adjacent medial definitive spine basal ganglia between 
them and to the oral definitive spine basal ganglion in their vicinity. In $(t)$, the red arrowheads indicate lateral neurites emerging from the radial nerve cords and innervating an oral definitive spine basal ganglion. (u-y) Neuroanatomy of the pedicellariae, in (u, v) transversal and (w-y) lateral views. In $(u-y)$, the white arrowheads indicate the valve nerves. In $(u, w, x)$, the white arrows mark the intervalve link nerves. In $(x, y)$, the red arrowheads point to connections between the pedicellaria nervous system and the basiepidermal nerve plexus. In (x), the red asterisks highlight the position of perikarya. AbdM, abductor muscle; AdM, adductor muscle; aDS, aboral definitive spine; DSBG, definitive spine basal ganglion; DSNrp, definitive spine neuropile; DSpk, definitive spine perikarya; mDS, medial definitive spine; oDS, oral definitive spine; PDN, podial distal neuropile; PP, primary podium; RNC, radial nerve cord; RingM, ring muscle; SN, spine nerve; SP, secondary podium.

\section{Figure 6: Neuroanatomy of Patiria miniata and Parastichopus parvimensis}

\section{post-metamorphic juveniles}

(a-q) Maximum intensity projections of confocal z-stacks for specimens co-labeled, in (a-c, $f-i, q)$, for acetylated $\alpha$-tubulin (AcTub) (neurons and cilia; yellow), F-actin (muscles; cyan), and DNA (nuclei; blue) or co-labeled, in (d, e, j-p), for acetylated $\alpha$-tubulin (AcTub) (neurons and cilia; yellow) and DNA (nuclei; blue). (a-c) Whole mount specimen of (a) P. miniata and (b, c) P. parvimensis postmetamorphic juveniles. In $(a, b)$, the specimens are in oral view. In $(c)$, the specimen is in left view with oral side up and ventral to the left. (d-q) Close-ups of (d, f-h, j-n) P. miniata and (e, i, o-q) P. parvimensis postmetamorphic juveniles. (d, e) Close-ups of the basiepidermal nerve plexus of (d) P. miniata and (e) P. parvimensis juveniles, on the aboral side. (f, g) Close-ups of a radial nerve cord of $P$. miniata juveniles. In (g), the red arrow highlights a lateral nerve innervating the nervous system of a secondary podium. (h, i) Close-ups of a secondary podium of (h) P. miniata and (i) $P$. parvimensis juveniles. In (h), the red arrowhead indicates a podia distal neuropile. $\ln (\mathrm{h}, \mathrm{i})$, the white arrowheads highlight the circumferential condensations present at the base of the papillae, and the yellow arrows mark the longitudinal condensations. (j, k) Close-ups of the right side of an interambulacrum of $P$. miniata juveniles at two different positions along the oral-aboral axis, $(k)$ being the most aboral. In $(j, k)$, the green arrowhead marks lateral nerves projecting from the 
adjacent radial nerve cord and innervating the asteroid arm spines. In (j), the yellow arrowheads indicate thin neurites spreading within the spine shafts. In $(k)$, the cyan arrowhead marks the spinefree zone of an interambulacrum, and the red arrows point to the same lateral nerve issued from the adjacent radial nerve cord and innervating both the spine arms and the spine-free zone of the interambulacrum. (l) Close-up of the spine-free zone of an interambulacrum of $P$. miniata juveniles. (m) Close-up of the webbed combs of two different interambulacra separated by a primary podium in $P$. miniata juveniles. $\ln (\mathrm{l}, \mathrm{m})$, the white arrowheads point to connections between lateral nerves and the basiepidermal nerve plexus. In $(\mathrm{m})$, the orange arrowhead indicates a direct connection between the neural structures of the webbed combs of two distinct interambulacra. (n) Close-up of a primary podium of $P$. miniata juveniles. $(o, p)$ Transversal views through (o) the ventral and $(p)$ a dorsal radial nerve cord of a $P$. parvimensis juvenile. In $(o, p)$, the dotted lines outline the radial nerve cords. In (o), the asterisk indicates the position of the canal. (q) Close-up of two primary podia of P. parvimensis juveniles. In (q), the pink arrows mark lateral nerves protruding from the circumoral nerve ring and innervating the primary podia nervous system. Bpl, basiepidermal nerve plexus; CNR, circumoral nerve ring; Eye, eye-spot; PP, primary podium; RM, radial muscle; RNC, radial nerve cord; SP, secondary podium; Spi, arm spine; VNC, ventral radial nerve cord.

\section{Supporting Information}

\section{Figure S1: External anatomy of Paracentrotus lividus early juveniles and close- ups of appendages}

(a) Light microscopy image of a 4-day-old early juvenile of $P$. lividus, in aboral view. Four days after metamorphosis, 4-day-old early juveniles are characterized, like their adult counterparts, by a spherical body with a central skeletal shell (the test) and several peripheral appendages, including the podia and the juvenile and definitive spines. $P$. lividus early juveniles exhibit pigment cells on the test and the spines. The podia are composed of a stretchy stem and a terminal, discoid papilla. (b-f) Scanning electron microscopy images of appendages of 4-day-old early juveniles of $P$. lividus. (b) Close-up of juvenile spines. Juvenile spines are immotile spines with either a triraradiate (yellow asterisks) or tetraradiate (cyan asterisks) tip. (c,d) Close-ups of pedicellariae. Pedicellariae are appendages composed of three movable jaws (white asterisks) that can either be (c) closed or (d) open. (e) Close-up of a definitive spine quartet. Within a quartet, the four definitive spines are morphologically indistinguishable from each other. Definitive spines are motile spines that are composed of a single pointed shaft, which displays an irregular surface, being regularly ornamented 
by spiky protrusions, and that is attached to the animal body through a basal tubercle (white asterisks). Within a quartet, the four spines are always successively arranged following a fixed pattern along the oral-aboral axis of the animal, with one spine towards the oral side, two spines in a medial position and one spine facing the aboral side. ( $f$ ) Close-up of a group of podia. Each group of podia includes a central, long primary podium and underneath it two smaller secondary podia. The external morphology of both podia types is similar. They are composed of a flexible stem that can extend and retract and that terminates by a discoid, flattened papilla located away from the body. Below the primary podium, we also often observed the presence of an emerging third secondary podium and of a growing sphaeridium, which in the adult forms a presumptive balance organ (Märkel et al., 1992). Abbreviations: aDS, aboral definitive spine; DS, definitive spine; JS, juvenile spine; $\mathrm{mDS}$, medial definitive spine; oDS, oral definitive spine; $\mathrm{PP}$, primary podium; $\mathrm{SP}$, secondary podium; SP', growing secondary podium; Sph, sphaeridia; TP, terminal papilla

\section{Figure S2: Close-ups of the endoskeleton of Paracentrotus lividus early juveniles}

(a-I) Maximum intensity projections of confocal z-stacks for specimens co-labeled for MSP-130 (endoskeleton; red) and DNA (nuclei; blue), except for (j), which is only labeled for MSP-130. (a-c) Close-ups of (a) a juvenile and $(b, c)$ a definitive spine, which are in $(a, b)$ lateral and (c) transversal views. In $(a, b)$, the insets show a computed transversal view of the corresponding spines. In $(a-c)$, the transversal views reveal the particular organization of the spine endoskeleton, which is composed of skeletal strands (white asterisks) that extend all along the shaft and alternate with interstitial tissue. Of note, there are four skeletal strands in juvenile spines (a) and six skeletal strands in definitive spines $(b, c)$, which supports the specific hexaradiate structure previously reported for the latter (Gosselin and Jangoux, 1998). In definitive spines, the skeletal strands further converge towards a central, skeleton-free, porous region (cyan asterisk in c), the medulla, which extends all along the shaft. (d,e) Close-ups of pedicellariae that can either be (d) closed or (e) open. In (d,e), the dotted lines demarcate the three movable jaws of the pedicellariae and the asterisks point to the stem. Note that only the stem of the pedicellariae is immunoreactive for MSP-130, which suggests that the calcite ossicles of the jaws must be formed by a different set of proteins. (f-h) Close-ups of $(f, g)$ a primary podium and $(h)$ a secondary podium, which are in $(f)$ lateral and $(g, h)$ transversal views. Primary and secondary podia both contain a single skeletal element (arrowhead in $f$ ), i.e., a flat disk located within the papilla and positioned perpendicular to the stem. Here, we named this structure the skeletal disk. The skeletal disks of $(\mathrm{g})$ primary and $(\mathrm{h})$ secondary podia are similar in size, and they both exhibit a central, skeleton-free pore (white asterisks). However, they are structurally different. The skeletal disks of $(\mathrm{g})$ primary podia are formed of a single ossicle, while those of $(\mathrm{h})$ secondary podia are formed by the fusion of three distinct ossicles, joined together at an angle of $120^{\circ}$ (Gosselin and Jangoux, 1998). In ( $h$ ), the dotted lines mark the boundary between the three 
ossicles constituting the secondary podium skeletal disk. (i-l) Close-ups of the pharyngeal area from (i) its most oral to (l) its most aboral side. (i) Close-up of the infracoronal region and the buccal plates. In (i), the dotted lines highlight the position of the five ambulacra, numbered in Latin numbers from 1 to $V$ after Lovén (1874), and the cyan asterisks mark the position of the buccal plate pores through which the buccal podia will emerge. Of note, the buccal plate pores display a specific "bbaba" pattern, which is defined based on their position on either the left "a" or the right " $b$ " side of the ambulacra, counting clockwise starting with ambulacrum 1 (David et al., 1995; Morris, 2009). Aboral to the buccal plates (i.e., in a more internal position within the juvenile), we further observed five bifid structures (yellow asterisks), which correspond to the jaws (or pyramids). (j) Cross-section through Aristotle's lantern allowing the visualization of the jaws. The dotted lines delineate two hemi-pyramids constituting a given jaw and the arrowhead points to a developing tooth. (k) Closeup of the most aboral part of the jaws. The arrowheads point to the developing teeth, each being located between two hemi-pyramids. (l) Close-up of the most aboral part of Aristotle's lantern. The white asterisks indicate the compasses, which are the most aboral skeletal elements of the masticatory apparatus. Abbreviations: BucPla, buccal plate; Phx, pharynx; TP, terminal papilla

\section{Figure S3: Close-ups of inner and muscular structures of Paracentrotus lividus early juveniles}

$(\mathrm{a}, \mathrm{b})$ Maximum intensity projections of confocal z-stacks for a 4-day-old early juvenile of $P$. lividus, in aboral view, illustrating the position of the digestive tract within the perivisceral cavity. In (a), the specimen is co-labeled for acetylated a-tubulin (AcTub) (neurons and cilia; yellow) and DNA (nuclei; blue). The blue and green dotted lines outline the digestive tract, with the stomach in blue and the intestine in green. The green asterisk marks the position of the esophagus (out of focus). In (b), the same juvenile than in (a) is shown but with only the acetylated a-tubulin staining, which is colorcoded along the oral-aboral axis of the animal to highlight the relative position of the different components of the digestive tract. (c-k) Maximum intensity projections of confocal z-stacks for specimens co-labeled, in (c,d,i), for F-actin (muscles; cyan) and DNA (nuclei; blue), labeled, in (e), for F-actin (muscles; cyan) or co-labeled, in ( $\mathrm{f}-\mathrm{h}, \mathrm{j}, \mathrm{k}$ ), for F-actin (muscles; cyan), MSP-130 (endoskeleton; red), and DNA (nuclei; blue). (c-g) Close-ups of muscles associated with the water vascular system. $(\mathrm{c}, \mathrm{d})$ Whole mount specimen in (c) lateral view with oral side down and (d) aboral view. The water vascular system includes a ring canal that forms a torus around the esophagus (white asterisks). Towards the supracoronal region, this canal extends through the axial complex, which is composed of several elements, including an axial coelom, a stone canal, and a hydropore (Ezhova et al., 2018; Ziegler et al., 2009). The stone canal and the hydropore constitute the unique connection of the water vascular system with the external environment. (e) Close-up of a radial canal. The white dotted line outlines the water vascular system elements associated with the radial canal. Towards the coronal region, the ring canal extends through five radial canals that spread along the ambulacra and innervate the podia. Each radial canal terminates in a primary podium and protrudes, on its lateral 
sides, lateral canals (yellow arrowheads) that individually connect to a secondary podium (out of focus) and an ampulla. Ampullae are small bags that are exclusively associated with secondary podia and enable them to extend and retract (Gosselin \& Jangoux, 1998; Märkel \& Röser, 1992; Nichols, 1972). In contrast, primary podiaare deprived of ampullae, their movement only depends on the coordinated extension and retraction of the five primary podia (Gosselin \& Jangoux, 1998). (f,g) Close-ups of $(f)$ a primary podium in lateral view and $(\mathrm{g})$ a secondary podium in transversal view. Primary and secondary podia are characterized by a similar inner musculature, with longitudinal muscles running all along the stem, reaching up to the tip of the papilla, and extending through the central pore of the skeletal disk. (h,i) Close-ups of pedicellariae, which are in (h) lateral and (i) transversal views. In $(h, i)$, the arrowheads point to the large adductor muscles, located in distal position (i.e., away from the shell), and the arrows indicate the thin abductor muscles, in proximal position. Each of these muscle types includes three muscles, which are each attached to two jaws of the pedicellariae. This attachment hence confers to these muscles a peculiar triangular pattern. Altogether, these muscles are responsible for coordinating the opening and closing movements of the jaws of the pedicellariae (Peters \& Campbell, 1987). (j,k) Close-ups of the tubercle of a definitive spine in $(j)$ lateral and $(k)$ transversal views. The definitive spine tubercle is surrounded by several muscle strands that form what we called the ring muscle. These strands are attached, on one side, to the juvenile test and, on the other side, to the spine endoskeleton, thereby enabling the spine to move in every direction. Abbreviations: Amp, ampulla; AxCoe, axial coelom; AxComp, axial complex; LMF, longitudinal muscle fiber; PP, Primary podium; PPSD, primary podium skeletal disk; RaC, radial canal; RiC, ring canal; RingM, ring muscle; SC, stone canal; SPSD, secondary podium skeletal disk

\section{Figure $S_{4}$ : Close-ups of the muscular anatomy of Aristotle's lantern}

(a) 3D reconstruction of the spatial arrangement of the five main muscles of Aristotle's lantern along the oral-aboral axis of the animal. Each muscle type is color-coded. (b-m) Maximum intensity projections of confocal z-stacks for specimens labeled, in (b,d,f-h,j,l), for F-actin (muscles; cyan), colabeled, in ( $k$ ), for F-actin (muscles; cyan) and DNA (nuclei; blue) or co-labeled, in (c,e,i,m), for Factin (muscles; cyan), MSP-130 (endoskeleton; red), and DNA (nuclei; blue). (b-c) Close-ups of the compass elevator and compass depressor muscles. (b) Close-up of the left half of Aristotle's lantern in lateral view to visualize both muscle types. The compass elevator and compass depressor muscles are unpaired muscles. Given the pentaradial organization of Aristotle's lantern, there are thus in total five compass elevator muscles and five compass depressor muscles. The compass elevator muscles are the most aboral muscles of the masticatory apparatus. They run parallel to the supracoronal and infracoronal regions and exhibit a characteristic pentagonal structure. The compass depressor muscles are, in contrast, arranged perpendicular to the compass elevator muscles. They are located on the side of the lantern and outside from it (i.e., away from the pharynx). (c) Close-up of the compass elevator muscles in aboral view, in a specimen co-stained for the endoskeleton. The compass elevator muscles stretch from one compass skeletal element to another and are required, 
together with the compass depressor muscles, to move these skeletal elements (Stauber, 1993; Ziegler et al., 2012). (d-g) Close-ups of the comminator muscles. (d) Cross-section through Aristotle's lantern illustrating the comminator muscles (white arrowheads), which correspond to the most prominent muscles of the masticatory apparatus. The comminator muscles are also unpaired muscles. They surround the pharyngeal area (outlined by the dotted line) and extend along each of the five ambulacra. Around the pharynx, the comminator muscles alternate with a muscle complex (white asterisks) that is composed of four distinct muscles, described in detail in (h-j). (e) Crosssection through Aristotle's lantern, in a specimen co-stained for the endoskeleton. The comminator muscles are positioned between the jaws (one of which is outlined by the dotted lines) and are connected to them (Ziegler et al., 2012). The white arrowhead highlights a developing tooth. (f) Close-up of a comminator muscle. The comminator muscles are composed of numerous, tightly packed, parallel strands, which spread along the oral-aboral axis of the lantern (see also b,j,k). On their pharyngeal side, the comminator muscles are further associated with two additional smaller muscles, the paired pharyngeal levators and the unpaired pharyngeal depressors. (g) Close-up of the pharyngeal area. The pharyngeal levator and pharyngeal depressor muscles stretch towards the mouth and reach the pharyngeal lips, which are themselves surrounded by circumferential muscle strands. (h-j) Close-ups of the retractor, protractor, postural, and dental promoter muscle complex. (h) Close-up of the complex in aboral view. The retractor, protractor, and postural muscles are long, thin, and paired muscles. In contrast, the dental promoter muscles are small and unpaired muscles, located at the midline between the retractors. (i) Close-up of the complex in aboral view, in a specimen co-stained for the endoskeleton. The protractor muscles are attached, on one side, to the top of the jaws (Ziegler et al., 2012) and, on the other side, to the test (yellow asterisks). The postural muscles are connected to the lateral sides of the two hemi-pyramids of a given jaw (cyan asterisks), and the dental promoter muscles lie at the junction between the two hemi-pyramids (cyan asterisks). (j) Close-up of the complex in lateral view. The retractor muscles are perpendicular to Aristotle's lantern. The protractor muscles are parallel to Aristotle's lantern, and thus to the comminator muscles. The postural muscles are a prolongation of the protractor muscles. $(\mathrm{k}-\mathrm{m})$ Close-ups of muscles present in the infracoronal region. (k) Close-up of Aristotle's lantern and the infracoronal region in lateral view. Several muscle fibers are detected below the comminator muscles, embedded in the peristome epidermis (white arrowhead). (l) Close-up of the muscle fibers in the peristome epidermis in oral view. Five pairs of radial muscle fibers extend strictly through the center of the peristome and are attached, on their proximal side, to the rim of the future mouth. In the center of the peristome, there are also circumferential muscles that surround the rim of the future mouth and extend up to the distal end of the radial muscle fibers. Around the circumferential muscles, additional muscle strands are detectable. They are short and straight. They are restricted to the remaining width of the peristome and extend parallel to the circumferential muscles, strictly along the interambulacra . $(\mathrm{m})$ Close-up of the radial muscle fibers of the peristome epidermis in oral view, in a specimen co-stained for the endoskeleton. On their distal end, the radial muscle fibers 
extend to the hemi-pyramids constituting the jaws (outlined by the white dotted lines).

Abbreviations: CirM, circumferential muscle; ComM, comminator muscle; Comp, compass; DepM, compass depressor muscle; DPM, dental promoter muscle; DS, definitive spine; ElvM, compass elevator muscle; MoR, Mouth rim; Phx, pharynx; PhxDM, pharyngeal depressor muscle; PhxLip, pharyngeal lip; PhxLM, pharyngeal levator muscle; ProM, protractor muscle; PstM, postural muscle; RadM, radial muscle; Ret $M$, retractor muscle; StM, straight muscle

\section{Figure S5: Distribution of serotonergic neurons in Paracentrotus lividus early juveniles and competent larvae}

(a-c) Maximum intensity projections of confocal z-stacks for specimens co-labeled for serotonin (5HT) (neurons; magenta), F-actin (muscles; cyan), and DNA (nuclei; blue). (a-b) Whole mount 4-dayold early juveniles of $P$. lividus in (a) oral and (b) aboral views. No specific serotonin-positive structures are detectable. (c) Whole mount competent larva of $P$. lividus in left side view, i.e., with the rudiment in oral view and the larval animal pole to the right. The white asterisk indicates the pharynx of the rudiment. The red arrowhead points to serotonergic neurons in the apical organ of the larva. The red arrow indicates a serotonergic nerve tract innervating the larval body from the larval apical organ. The yellow arrowheads highlight serotonergic neurons and neurites present in the larval ciliary band. Abbreviations: AO, apical organ; PP, primary podium; RingM, ring muscle

\section{Figure S6: Close-ups of the neuromuscular anatomy of Aristotle's lantern and the peristome epidermis in Paracentrotus lividus early juveniles}

(a-g) Maximum intensity projections of confocal z-stacks for specimens co-labeled for F-actin (muscles; cyan) and synaptotagmin-B (SytB) (neurons; green), except for (e), which is only labeled for synaptotagmin-B (SytB). In (a-d,f,g), the first column shows F-actin staining, the second column displays SytB staining, and the third column merges F-actin and SytB staining. In (e), a composite image of SytB staining is provided that highlights the relative oral (blue) versus aboral (yellow) position of the circumoral and the hyponeural nerve ring and their associated nerves bundles. (a-e) Close-ups of muscles and neural structures associated with Aristotle's lantern. In (a-a"), the asterisks highlight a dental promoter muscle and its co-localizing neurites. Note that these neurites are positioned at an angle of the hyponeural nerve ring(see also (e)). In (b-b"), the arrowheads point to two pharyngeal levator muscles and their associated neurites. In (c-c"), the arrows mark the retractor muscles and their related neurites, and the arrowheads highlight the protractor muscles and their co-localizing neurites. In (d-d"), the arrows point to the base of the retractor muscles and their associated neuropiles positioned in the vicinity of the circumoral nerve ring. In (e), the red arrowhead indicates a straight nerve bundle, positioned at the midline of a segment of the circumoral nerve ring (blue). Note that this nerve bundle extends in continuation of a nerve bundle that is located in a more aboral position, at the level of an angle of the hyponeural nerve ring 
(yellow), and that co-localizes with the dental promoter muscles (see also (a-a")). It remains unclear, however, whether the nerve bundles at the level of the circumoral nerve ring also innervate the dental promoter muscles. $(f-g)$ Close-ups of muscles and neural structures associated with the peristome epidermis. In ( $\left.f-f^{\prime}\right)$, the arrowheads mark the distal end of the radial muscle fibers, which co-localize with two neural clusters located at the base of the radial nerve cords. In (g-g"), the arrows highlight radial muscle fibers spreading throughout the peristome epidermis and their associated neurites. Abbreviations: CNR, circumoral nerve ring; HypNR, hyponeural nerve ring; Phx, pharynx

\section{Figure S7: Anatomical survey of Patiria miniata and Parastichopus parvimensis postmetamorphic juveniles}

(a-c) Postmetamorphic juveniles of $P$. miniata (30 days after metamorphosis). (d-f) Postmetamorphic juveniles of $P$. parvimensis (20 days after fertilization). (a-a",d) Light microscopy images. (b,c,e,f) Maximum intensity projections of confocal z-stacks for specimens co-labeled for F-actin (muscles; cyan) and DNA (nuclei; blue). (a) Whole-mount specimen of a $P$. miniata juvenile in oral view. Compared to $P$. lividus early juveniles, $P$. miniata juveniles have a relatively flat body, which renders their ambulacra (yellow dotted lines) perfectly perpendicular to the oral-aboral axis of the animal. Like in P. lividus, each $P$. miniata ambulacrum terminates with a primary podium, and secondary podia (pink asterisks) are distributed underneath the former on both the left and right side of the ambulacrum. Compared to the interambulacra of $P$. lividus, however, those of $P$. miniata are much larger. They also bear the main spines of the animal (i.e., the arm spines), which form, at each extremity of an interambulacrum, a webbed comb, composed of four to six flat spines. (a') Close-up of a $P$. miniata ambulacrum. The ambulacra of $P$. miniata feature an asteroid specificity, i.e., an eyespot (or optic cushion) (red arrowhead), that is located just below the stem of the primary podia (Petie et al., 2016). (a") Close-up of the aboral face (or supracoronal region) of a P. miniata juvenile. The white arrowheads mark some of the juvenile spines observed on the supracoronal region. At the stage investigated, no pedicellariae were observed. (d) Whole mount specimen of a postmetamorphic juvenile of $P$. parvimensis, in left side view, with oral side up and ventral to the left. In holothuroids, larval metamorphosis is initiated much earlier after fertilization than in other echinoderm classes and is a more gradual process (Morgan, 2008; Smiley, 1986). These features thus render an individual 20 days after fertilization, a so-called pentactula larva, morphologically equivalent and thus comparable to a P. lividus early juvenile (Smirnov, 2015). Compared to $P$. lividus early juveniles, postmetamorphic $P$. parvimensis individuals have a tubular-shape, with five tentacles (red asterisks), on their oral side, distributed around the mouth, and one foot (pink asterisk), on the opposite side, used to attach to the substrate. The five oral tentacles correspond to the five primary podia of the juvenile, and the aboral foot is its unique secondary podium (Smiley, 1986). This secondary podium further defines the ventral side of the juvenile. The holothuroid body plan is indeed characterized by 
a bilateral symmetry that has secondarily been reacquired in the course of evolution (Kerr \& Kim, 1999). Apart from the podia, P. parvimensis juveniles, however, do not feature any other appendages, neither spines nor pedicellariae. (b-f) Muscular anatomy of postmetamorphic juveniles of $(b, c) P$. miniata and (e,f) P. parvimensis. (b-f) Whole mount specimens in (b,e) oral, (c) aboral, and (f) left view with oral side up and ventral to the left. Both $P$. miniata and $P$. parvimensis juveniles are characterized by an elaborated muscular system. Like in P. lividus early juveniles, in both P. miniata and $P$. parvimensis juveniles, the muscles are chiefly associated with the masticatory apparatus, the water vascular system, and the appendages. As one example, similar to the situation in P. lividus early juveniles, the podia of $P$. miniata and $P$. parvimensis display long, longitudinal muscles that extend throughout the stem. In contrast, unlike the secondary podia of $P$. lividus and $P$. miniata, that of $P$. parvimensis does not feature any ampulla at its base. In $P$. miniata juveniles, there are further long, longitudinal arm muscles, along each ambulacrum, for which no equivalent was found in $P$. lividus or P. parvimensis. Lastly, the spines of $P$. miniata, located at the extremity of each interambulacrum, are characterized by small muscles, which appear much simpler in structure than the ring muscles associated with the definitive spines of $P$. lividus. Abbreviations: Amp, ampulla; LAM, longitudinal arm muscle; Phx, pharynx; PP, primary podium; RM, radial muscle; SP, secondary podium; Spi, arm spine; St, stomach; VM, ventral muscle

\section{References for supplementary information material}

David, B., Mooi, R., \& Telford, M. (1995). The ontogenetic basis of Lovén's Rule clarifies homologies of the echinoid peristome. In R. Emson, A. B. Smith, \& A. C. Campbell (Eds.), Echinoderm Research 1995, 155-164. Rotterdam: AA Balkema.

Ezhova, O. V., Malakhov, V. V., \& Egorova, E. A. (2018). Axial complex and associated structures of the sea urchin Strongylocentrotus pallidus (Sars, G.O. 1871) (Echinodermata: Echinoidea). Journal of Morphology, 279(6), 792-808.

Gosselin, P., \& Jangoux, M. (1998). From competent larva to exotrophic juvenile: A morphofunctional study of the perimetamorphic period of Paracentrotus lividus (Echinodermata, Echinoida). Zoomorphology, 118(1), 31-43.

Kerr, A. M., \& Kim, J. (1999). Bi-penta-bi-decaradial symmetry: A review of evolutionary and developmental trends in holothuroidea (Echinodermata). The Journal of Experimental Zoology, 285(2), 93-103.

Lovén, S. (1874). Études sur les échinoïdées. Kongelige Svenska Vetenskaps-Akademiens Handlingar, 11, 1-91. Stockholm: P. A. Norstedt \& Söner.

Märkel, K., Mackenstedt, U., \& Röser, U. (1992). The sphaeridia of sea urchins: Ultrastructure and supposed function (Echinodermata, Echinoida). Zoomorphology, 112(1), $1-10$.

Märkel, K., \& Röser, U. (1992). Functional anatomy of the valves in the ambulacral system of sea urchins (Echinodermata, Echinoida). Zoomorphology, 111(3), 179-192. 
Morgan, A. (2008). Metamorphosis in larvae of the temperate sea cucumber Australositchopus mollis. Invertebrate Reproduction \& Development, 51(3), 127-143.

Morris, V. B. (2009). On the sites of secondary podia formation in a juvenile echinoid: Growth of the body types in echinoderms. Development Genes and Evolution, 219(11), 597608.

Nichols, D. (1972). The water-vascular system in living and fossil echinoderms. Palaeontology, 15(4), 519-538.

Peters, B. H., \& Campbell, A. C. (1987). Morphology of the nervous and muscular systems in the heads of pedicellariae from the sea urchin Echinus esculentus L. Journal of Morphology, 193(1), 35-51.

Smiley, S. (1986). Metamorphosis of Stichopus californicus (Echinodermata: Holothuroidea) and its phylogenetic implications. Biological Bulletin, 171(3), 611-631.

Smirnov, A. V. (2015). Paedomorphosis and heterochrony in the origin and evolution of the class holothuroidea. Paleontological Journal, 49(14), 1597-1615.

Stauber, M. (1993). The lantern of Aristotle: Organization of its coelom and origin of its muscles (Echinodermata, Echinoida). Zoomorphology, 113(2), 137-151.

Ziegler, A., Faber, C., \& Bartolomaeus, T. (2009). Comparative morphology of the axial complex and interdependence of internal organ systems in sea urchins (Echinodermata: Echinoidea). Frontiers in Zoology, 6(1), 10.

Ziegler, A., Schröder, L., Ogurreck, M., Faber, C., \& Stach, T. (2012). Evolution of a novel muscle design in sea urchins (Echinodermata: Echinoidea). PLOS ONE, 7(5), e37520. 